\title{
A Multiple-Window Video Embedding Transcoder Based on H.264/AVC Standard
}

\author{
Chih-Hung Li, Chung-Neng Wang, and Tihao Chiang \\ Department of Electronics Engineering, National Chiao-Tung University, 1001 Ta-Hsueh Road, Hsinchu 30010, Taiwan
}

Received 6 September 2006; Accepted 26 April 2007

Recommended by Alex Kot

This paper proposes a low-complexity multiple-window video embedding transcoder (MW-VET) based on H.264/AVC standard for various applications that require video embedding services including picture-in-picture (PIP), multichannel mosaic, screensplit, pay-per-view, channel browsing, commercials and logo insertion, and other visual information embedding services. The MW-VET embeds multiple foreground pictures at macroblock-aligned positions. It improves the transcoding speed with three block level adaptive techniques including slice group based transcoding (SGT), reduced frame memory transcoder (RFMT), and syntax level bypassing (SLB). The SGT utilizes prediction from the slice-aligned data partitions in the original bitstreams such that the transcoder simply merges the bitstreams by parsing. When the prediction comes from the newly covered area without slice-group data partitions, the pixels at the affected macroblocks are transcoded with the RFMT based on the concept of partial reencoding to minimize the number of refined blocks. The RFMT employs motion vector remapping (MVR) and intra mode switching (IMS) to handle intercoded blocks and intracoded blocks, respectively. The pixels outside the macroblocks that are affected by newly covered reference frame are transcoded by the SLB. Experimental results show that, as compared to the cascaded pixel domain transcoder (CPDT) with the highest complexity, our MW-VET can significantly reduce the processing complexity by 25 times and retain the rate-distortion performance close to the CPDT. At certain bit rates, the MW-VET can achieve up to $1.5 \mathrm{~dB}$ quality improvement in peak signal-to-noise-ratio (PSNR).

Copyright (c) 2007 Chih-Hung Li et al. This is an open access article distributed under the Creative Commons Attribution License, which permits unrestricted use, distribution, and reproduction in any medium, provided the original work is properly cited.

\section{INTRODUCTION}

Video information embedding technique is essential to several multimedia applications such as picture-in-picture (PIP), multichannel mosaic, screen-split, pay-per-view, channel browsing, commercials and logo insertion, and other visual information embedding services. With the superior coding performance and network friendliness, H.264/AVC [1] is regarded as a future multimedia standard for service providers to deliver digital video contents over local access network (LAN), digital subscriber line (DSL), integrated services digital network (ISDN), and third generation (3G) mobile systems [2]. Particularly, the next generation Internet protocol television service (IPTV) could be realized with H.264/AVC over very-high-bit-rate DSL (VDSL), which can support higher transmission rates up to $52 \mathrm{Mbps}$ [3]. The service with high transmission rate facilitates the development of video services with more functionalities and higher interactivity for video over DSL applications. For video embedding applications, the video em- bedding transcoder (VET) is essential to deliver multiplewindow video services over one transmission channel.

The VET functionality can be realized at the client side where multiple sets of tuners and video decoders acquire video content of multiple channels for one frame. The content delivery side sends all the bitstreams of selected channels to the client while the client side reconstructs the pixels with an array of decoders in parallel and then re-composes the pixels into single frame in the pixel domain at the receivers. Each receiver needs $N$ decoders running with a powerful picture composition tool to tile the varying size pictures from $N$ channels. Thus, the overall cost is increased as $N$ is increased. To reduce the cost of the VET service, fast pixel composition and less memory access can be achieved based on the architecture design [4-16]. To realize the VET feature at the client side, the key issues are inefficient bandwidth utilization and high hardware complexity that hinders the multiple-window embedding applications deployment.

To increase the bandwidth efficiency and reduce hardware complexity, the VET functionality is realized at the 
server/studio side to deliver selected video contents that are encapsulated as one bitstream. The challenges are to simultaneously maintain the best picture quality after transcoding, to increase the picture insertion flexibility, to minimize the archival space of bitstreams, and to reduce hardware complexity. To optimize rate-distortion (R-D) performance, the bits of the newly covered blocks at the background picture are replaced by the bits of the blocks at the foreground pictures. To increase the flexibility of picture insertion, the foreground pictures are inserted at the macroblock boundaries of processing units. To minimize the bitstream storage space, H.264/AVC coding standard is adopted as the target format. To decrease the hardware complexity, a low-complexity algorithm for composition is needed. Therefore, we proposed a fast H.264/AVC-based multiple-window VET (MW-VET), which encapsulates on-the-fly multiple channels of video content with a set of precompressed bitstreams into one bitstream before transmission.

To transmit the video contents via the unitary channel, the MW-VET embeds downsized video frames into another frame with a specified resolution as the foreground areas. It can provide preview frames or thumbnail frames by tiling a two-dimensional array of video frames from multiple television channels simultaneously. With the MW-VET, users can acquire multiple-channel video contents simultaneously. Moreover, the MW-VET bitstreams are compliant to H.264/AVC and it can facilitate the multiple-window video playback in a way transparent to the decoder at the client side.

For real-time applications, video transcoding should retain R-D performance with the lowest complexity, minimal delay, and the smallest memory requirement [17]. Particularly, the MW-VET should maintain good quality after multigeneration transcoding that may aggravate the quality degradation. An efficient VET transcoder is critical to address the issue of quality loss. For complexity reduction, existing approaches [18-21] convert the bitstreams that are of MPEG-2 standard in the transform domain. Application of the existing transcoding techniques to H.264/AVC is not feasible since the advanced coding tools including in-the-loop deblocking filter, directional spatial prediction, and 6-tap subpixel interpolation all operate in the pixel domain. Consequently, the transform domain techniques have higher complexity as compared to the spatial domain techniques.

To maintain transcoded picture quality and to reduce the overall complexity, we present three transcoding techniques: (1) slice-group-based transcoding (SGT), (2) reduced frame memory transcoding (RFMT), and (3) syntax level bypassing (SLB). The application of each transcoding technique depends on the data partitions of the archived bitstreams and the paths of error propagation. For slice-aligned data partitions, the SGT that composes the VET bitstreams at the bitstream level can provide the highest throughput. For regionaligned data partitions, the RFMT efficiently refines the prediction mismatch and increases throughput while maintaining better R-D performance. For the blocks that are not affected by the drift error, the SLB de-multiplexes and multiplexes the bitstreams into a VET bitstream at the bitstream level. As the foreground bitstreams are encoded as full resolution, a downsizing transcoding [22-24] is needed prior to the VET transcoding. The spatial resolution adaptation transcoders have been widely investigated in the literatures and are not studied herein.

Our experimental results show that the MW-VET architecture significantly reduces processing complexity by 25 times with similar or even higher R-D performance as compared to the conventional cascaded pixel domain transcoder (CPDT). The CPDT cascades several decoders and an encoder for video embedding transcoding. It offers drift free performance with the highest computational cost. With the fast transcoding techniques, the MW-VET can achieve up to $1.5 \mathrm{~dB}$ quality improvement in peak signal-to-noise ratio (PSNR).

The rest of this paper is organized as follows: Section 2 describes the issues for the video embedding transcoding. Section 3 reviews the related works and Section 4 describes our H.264/AVC-based MW-VET. Section 5 shows the simulation results and Section 6 gives the conclusion.

\section{PROBLEM STATEMENT}

Transcoding process could be viewed as the modification process of incoming residue according to the changes in the prediction. As shown in Figure 1(a), the output of transcoding is represented by

$$
R_{n}^{\prime}=\mathrm{Q}\left[\mathrm{HT}\left(r_{n}^{\prime}\right)\right]=\mathrm{Q}\left[\mathrm{HT}\left(\overline{r_{n}}+\operatorname{Pred}_{1}\left(\overline{y_{n}}\right)-\operatorname{Pred}_{2}\left(\overline{y_{n}^{\prime}}\right)\right)\right],
$$

where the symbols HT and Q indicate an integer transformation and quantization, respectively. The symbols $\overline{r_{n}}$ and $r_{n}^{\prime}$ denote the residue before and after the transcoding. The symbols $\operatorname{Pred}_{1}\left(\overline{y_{n}}\right)$ and $\operatorname{Pred}_{2}\left(\overline{y_{n}^{\prime}}\right)$ represent the predictions from the reference data $\overline{y_{n}}$ and $\overline{y_{n}^{\prime}}$, respectively. In this paper, we use the symbol "bar" above the variables to denote the reconstructed values after decoding and the symbol "prime" to denote the refined values after transcoding. The suffix of each variable represents the index of block. The process to embed the foreground videos onto the background can incur drift error in the prediction loop since the reference frames at the decoder and the encoder are not synchronized.

When the predictions before and after the transcoding are identical, Figure 1(a) can be simplified to Figure 1(b). The quantized data $\overline{r_{n}}$ has no further quantization distortion with the same quantization step. Thus, the transcoded bitstream has almost identical R-D performance with the original bitstream as represented in:

$$
P_{d} \cdot P_{e} \cdot \overline{r_{n}}=\operatorname{IHT}\left\{\operatorname{IQ}\left\{\mathrm{Q}\left[\mathrm{HT}\left(\overline{r_{n}}\right)\right]\right\}\right\}=\overline{r_{n}},
$$

where the symbol $P_{e}$ denotes the encoding process from the pixel domain to the transform domain. The symbol $P_{d}$ denotes the decoding process from the transform domain back to the pixel domain. The symbols IHT and DQ mean an inverse integer transformation and dequantization, respectively.

By (2), the transcoding process in Figure 1(b) can be further simplified to that in Figure 1(c), where the data of the 


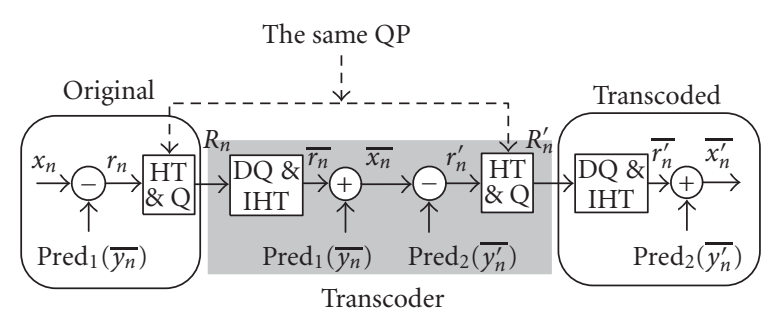

(a)

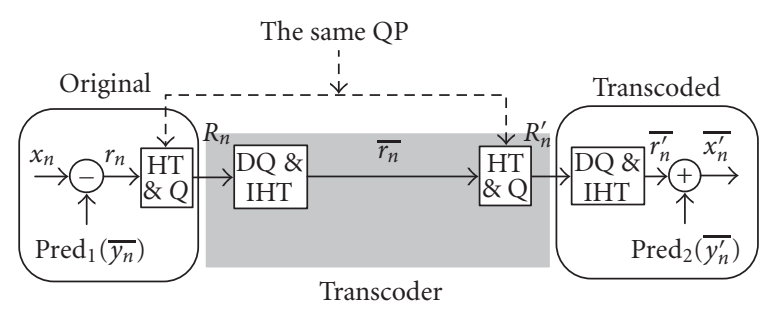

(b)

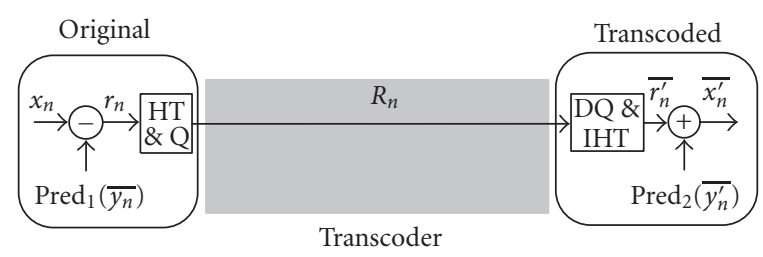

(c)

Figure 1: Illustration of a novel transcoder: (a) the simplified transcoding process, (b) the simplified transcoder when the prediction blocks are the same, (c) the fast transcoder that can bypass the input transform coefficients.

original bitstreams can be bypassed without any modification. It leads to a transcoding scheme with the highest R-D performance and the lowest complexity.

Video transcoding is intended to maximize R-D performance with the lowest complexity. Therefore, the remaining issue is to transcode efficiently the incoming data such that picture quality is maximized with the lowest complexity. Specifically, the incoming data are refined only when the reference pixels are modified to alleviate the propagation error. To reduce computational cycles and preserve picture quality, the residue data with identical reference pixels are bypassed.

\section{RELATED WORKS ON PICTURE-IN-PICTURE TRANSCODING}

Depending on which domain is used to transcode, the transcoders can be classified as either pixel domain or transform domain approaches.

\subsection{Cascaded pixel domain transcoder}

The cascaded pixel domain transcoder (CPDT) cascades multiple decoders, a pixel domain composer, and an encoder, as shown in Figure 2. It decompresses multiple bitstreams, composes the decoded pixels into one picture, and recom-

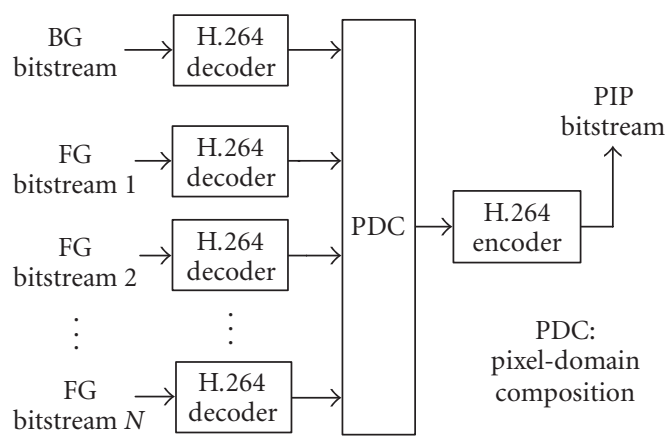

Figure 2: Architecture of the CPDT.

presses the picture into a new bitstream. The reencoding process of CPDT can avoid drift errors from propagating to the whole group of pictures.

However, the CPDT suffers from noticeable visual quality degradation and high complexity. Specifically, the requantization process decreases quality of the original bitstreams. The quality degradation exacerbates especially when the foreground pictures are inserted at different time using the CPDT with multiple iterations. In addition, the reencoding makes the significant complexity increase of the CPDT too costly for real-time video content delivery. The complexity and memory requirement of the CPDT could be reduced with fast algorithms that remove inverse transformation, motion compensation, and motion estimation.

\subsection{DCT domain transcoding with motion vector remapping}

The inverse transformation can be eliminated with the discrete cosine transform (DCT) domain inverse motion compensation (IMC) approach proposed by Chang et al. [18-20] for the MPEG-2 transcoders. The matrix translation manipulations are used to extract a DCT block that is not aligned to the boundaries of $8 \times 8$ blocks in the DCT domain. Chang's approach could achieve $10 \%$ to $30 \%$ speedup over the CPDT. There are other algorithms to speed up the DCT domain IMC in [25-27].

The motion estimation can be eliminated with motion vector remapping (MVR) where the new motion vectors are obtained by examining only two most likely candidate motion vectors located at the edges outside the foreground picture. It simplifies the reencoding process with negligible picture quality degradation.

\subsection{DCT domain transcoding with backtracking}

A DCT domain transcoder based on a backtracking process is proposed by Yu and Nahrstedt [21] to further improve the transcoding throughput. The backtracking process finds the affected macroblocks (MBs) of the background pictures in the motion prediction loop. Since only a small percentage of the MBs at the background are affected, only the damaged MBs are fixed and the unaffected MBs are bypassed. 
In practice, for most effective backtracking, the future motion prediction path of each affected MB needs to be analyzed and stored in advance. To construct the motion prediction chains, Chang et al. [18-20] completely reconstructs all the refined reference frames in the DCT domain for each group-of-picture (GOP). With the motion prediction chains, the transcoder decodes minimum number of MBs to render the correct video contents. The speedup of motion compensation is up to $90 \%$ at the cost of the buffering delay of the transcoder for one GOP period. The impact of the delay on the real-time applications depends on the length of a GOP in the original bitstream.

However, the backtracking method has no use for the H.264/AVC-based transcoder due to the deblocking filter, the directional spatial prediction, and interpolation filter. In addition, to track the prediction paths of H.264/AVC bitstreams, almost $100 \%$ of the blocks need decoding, which is over the $10 \%$ reported in [21]. Thus, the expected complexity reduction is limited. Furthermore, it introduces an extra delay of one GOP period.

In summary, to speed up the CPDT, there are many fast algorithms to manipulate the incoming bitstreams in the transform domain. However, this is not the case for the H.264/AVC standard. To our best knowledge, all the state-ofthe-art transcoding schemes with H.264 as input bitstream format perform the fast algorithms in the pixel domain [2836]. There are several reasons to manifest the necessity of pixel domain manipulation. As shown in the appendix the pixel domain transcoder actually takes less complexity than the transform domain transcoder. The detail derivations are given in the appendix for brevity. In addition, the transform domain manipulation introduces drift because the motion compensation is based on the filtered pixels which are the output of the in-the-loop deblocking filter. The filtering operation is defined in the pixel domain and cannot be performed in the transform domain due to its nonlinear operations [28-30]. As a result, the transform domain transcoder for the H.264/AVC standard typically leads to an unacceptable level of error as shown in [37]. Therefore, we conclude that the spatial domain technique is a more realistic approach for H.264/AVC-based transcoding. To resolve issues of low computational cost, less drift error, and small memory bandwidth, we present an H.264/AVC-based transcoder in the spatial domain.

\section{LOW-COMPLEXITY MULTIPLE-WINDOW VIDEO EMBEDDING TRANSCODER (MW-VET)}

For real-time delivery of high quality video bitstreams, our goal is to build the bitstreams with the picture quality close to that of the original bitstream using smallest complexity. To minimize cost and memory requirement and retain the best picture quality, we present a low-complexity multiple window video embedding transcoder (MW-VET) suitable for both interactive and noninteractive applications. In Table 1, we list all the symbol definitions used in the proposed architectures.
TABLE 1: Symbol definitions.

\begin{tabular}{l|l}
\hline Symbol & Meaning \\
\hline CAVLD & Content adaptive variable length decoding \\
CAVLC & Content adaptive variable length coding \\
LB & Line buffer \\
FM & Frame memory \\
DB & Deblocking filter \\
IP & Intra prediction \\
MC & Motion compensation \\
ME & Motion estimation \\
HT \& Q & Integer transform and quantization \\
DQ \& IHT & Dequantization and inverse integer transform \\
PDC & Pixel domain composition \\
RDO MD & Rate-distortion optimized mode decision \\
MUX & Multiplexer (syntax element selector) \\
\hline
\end{tabular}

\subsection{Rationale}

To embed foreground pictures as multiple windows to one background picture, the MW-VET inserts the foreground pictures at MB-aligned positions. To minimize complexity, it uses several approaches including slicegroup-based transcoding (SGT), reduced-frame-memory transcoder (RFMT), and syntax level bypassing (SLB) to adapt the prediction schemes compliant with the H.264/AVC standard. As the prediction is applied to the slice-aligned data partitions within the original bitstreams, the SGT merges the original bitstreams into one bitstream by parsing and concatenation leading to a fast transcoding. For noninteractive services, the SGT can provide the highest transcoding throughput if the original bitstreams are coded with the slicealigned data partitions.

When the prediction is applied to the region-aligned data partitions, the specified pixels at the background picture are replaced by the pixels of the foreground pictures. For the pixels in the affected MBs, the RFMT can minimize the total number of refined blocks by partially reencoding only those MBs. The RFMT employs both motion vector remapping (MVR) for intercoded blocks and intramode switching (IMS) for intracoded blocks, respectively. The pixels within the unaffected MBs are transcoded by the SLB that passes the syntax elements from the original bitstreams to the transcoded bitstream.

Based on the occurrence of modified reference pixels at the prediction loop, the MBs are classified into three types: $w$-MB, $p-\mathrm{MB}$, and $n-\mathrm{MB}$. As shown in Figure 3, the small rectangle denotes the foreground picture $(\mathrm{FG})$ and the large rectangle denotes the background picture (BG). Each small square within the rectangle represents one MB. The $w$-MBs represent the blocks whose reference samples are entirely or partially replaced by the newly inserted pictures. The $p$-MBs represent the blocks whose reference pixels are composed of the pixels at $w$-MBs. The remaining MBs of the background pictures are denoted as $n$-MBs for the unaffected MBs. We observe that most of the MBs within the processing picture are $p$-MBs and only a small percentage of MBs are $w$-MBs. As 


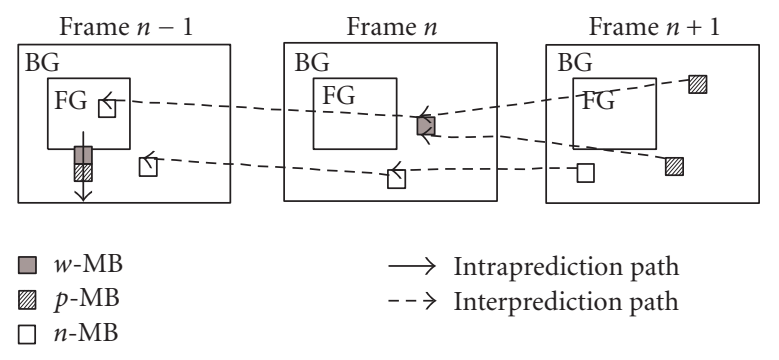

FIGURE 3: Illustration of the wrong reference problem.

for $w$-MBs, the coding modes or motion vectors of the original bitstream are modified to fix the wrong reference problem. For the $p-\mathrm{MBs}$, the wrong reference problem is inherited from the $w$-MBs. Thus, the coding modes and motion vectors are refined for each $p$-MB. All $n$-MBs' information in the original bitstream can be bypassed because the predictors before and after transcoding are identical.

\subsection{Slice-group-based transcoding}

The slice-group-based transcoding (SGT) is used when the prediction within the original bitstream of background picture uses the slice-aligned data partitions [38]. Based on the slice-aligned data partitions, the SGT operates at the bitstream level to provide the highest throughput with the lowest complexity. The rationale is that H.264/AVC defines a set of MBs to the slice group map types according to the adaptive data partition [1]. The concept of slice group is to separate the picture into isolated regions to prevent error propagation from leading error resiliency and random access. Each slice is regarded as an isolated region as defined in H.264/AVC standard. For each region, the encoder performs the prediction and filtering processes without referring to the pixels of the other regions.

For the video embedding feature using static slice groups, the large window denotes a background slice and the embedded small windows denote foreground slices. After video embedding transcoding, all the slices are encoded separately at the slice level and encapsulated to one bitstream at the slice level. Based on archived H.264/AVC bitstreams with the slice groups, a VET can replace the syntax elements of MBs in the foreground slices with the syntax elements of other bitstreams with identical spatial resolutions. Therefore, all the syntax elements are directly forwarded as is to the final bitstream via an entropy coder. In conclusion, the SGT is effective for noninteractive applications with multiple static windows.

\subsection{Reduced frame memory transcoding}

Based on the partially reencoding techniques, the initial RFMT architecture is shown in Figure 4. After decoding all the bitstreams into pixel domain with multiple H.264/AVC decoders and composing all the decoded pictures into one frame by the PDC, the reencoder side only refines the residue of the affected MBs rather than reencoding all the decoded pixels as the CPDT architecture. For those unaffected MBs, the syntax elements are bypassed from each CAVLD and are sent to the MUX which selects the corresponding syntax elements based on the PIP scenario. Lastly, the CAVLC encapsulates all the reused syntax elements and the new syntax elements of refined blocks into the transcoded bitstream.

To increase the throughput, the R-D optimized mode decision and motion vector reestimation within the reencoder side of Figure 4 are replaced with the intramode switching (IMS) and motion vector remapping (MVR) as shown in Figure 5 [39]. Specifically, the reencoder as enclosed by the dashed line stores the decoded pixels into the FM. Then, the MVR and IMS modules retrieve the intra modes and the motion vectors from the original bitstreams to predict the characteristics of motion and the spatial correlation of the source. With such information, we examine only a subset of possible motion vectors and intra modes to speed up the refinement process. According to the refined motion vectors and coding modes, the MC and IP modules perform motion compensation and intraprediction from the data in the FM and LB. The reconstruction loop including HT, Q, DQ, IHT, and DB generates the reconstructed data of the refined blocks which are further stored in the FM to avoid the drift during the transcoding. In conclusion, other than the IMS and MVR modules all the modules in Figure 5 are the same as those in Figure 4.

To decouple the dependency between the foreground and the background, there is an encoding constraint for the foreground bitstream that the unrestricted motion vectors and the intra-DC modes are not used for the blocks at the first column or the first row. When the foreground video is from an archived bitstream or an encoder of live video, the unrestricted motion vectors and the intra DC mode can be modified and the loss of R-D performance is negligible according to our experiment. Particularly, we rescale the DC coefficient of the first DC block within an intracoded frame based on the neighboring reconstructed pixels in the background. Except the first block, the foreground bitstreams can be multiplexed directly into the transcoded bitstream.

With the constrained foreground bitstreams, the final architecture of the MW-VET is simplified as shown in Figure 6. The highly efficient MW-VET adopts only the content adaptive variable length decoding (CAVLD) for the foreground bitstreams and uses one shared frame memory for the background bitstream. At first, two frame memories are dedicated for the decoder and the reencoder in Figure 5 to store the decoded pixels and the reconstructed pixels, respectively. However, the decoded data of affected blocks are no longer useful and could be replaced with the reconstructed pixels after the refinement. Therefore, we use a shared frame memory to buffer the reference pixels for both the decoding and reencoding process. Specifically, the operation of the transcoder begins with the decoding by the CAVLD. The MC and the IP modules in the left-hand side use the original motion vectors and intra modes to decode the source bitstream into pixels stored in the FM and used for the coefficient refinement. On the other hands, the MC and the IP modules in the righthand side use the refined motion vectors and intra modes to 


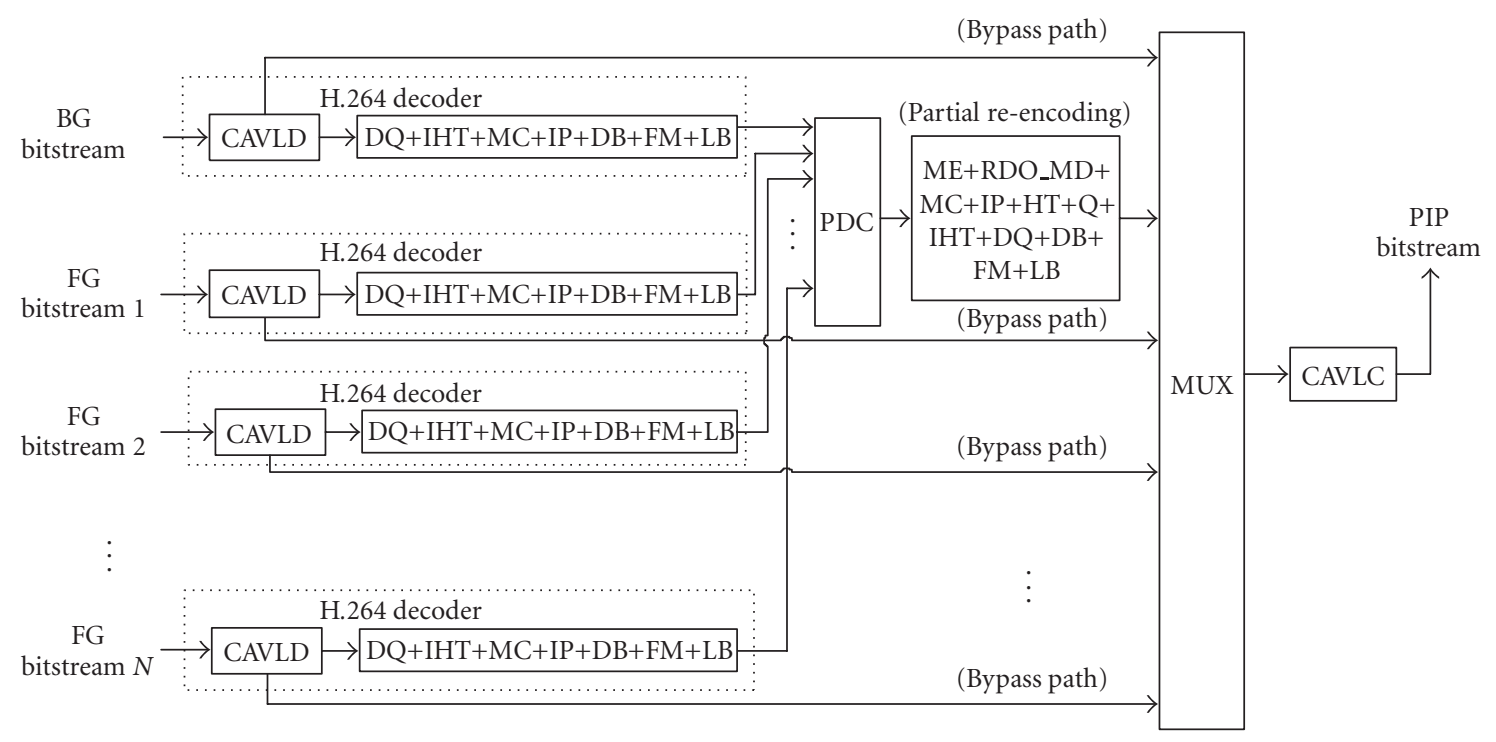

FIGURE 4: Initial architecture of RFMT with RDO refinement based on the partially reencoding.

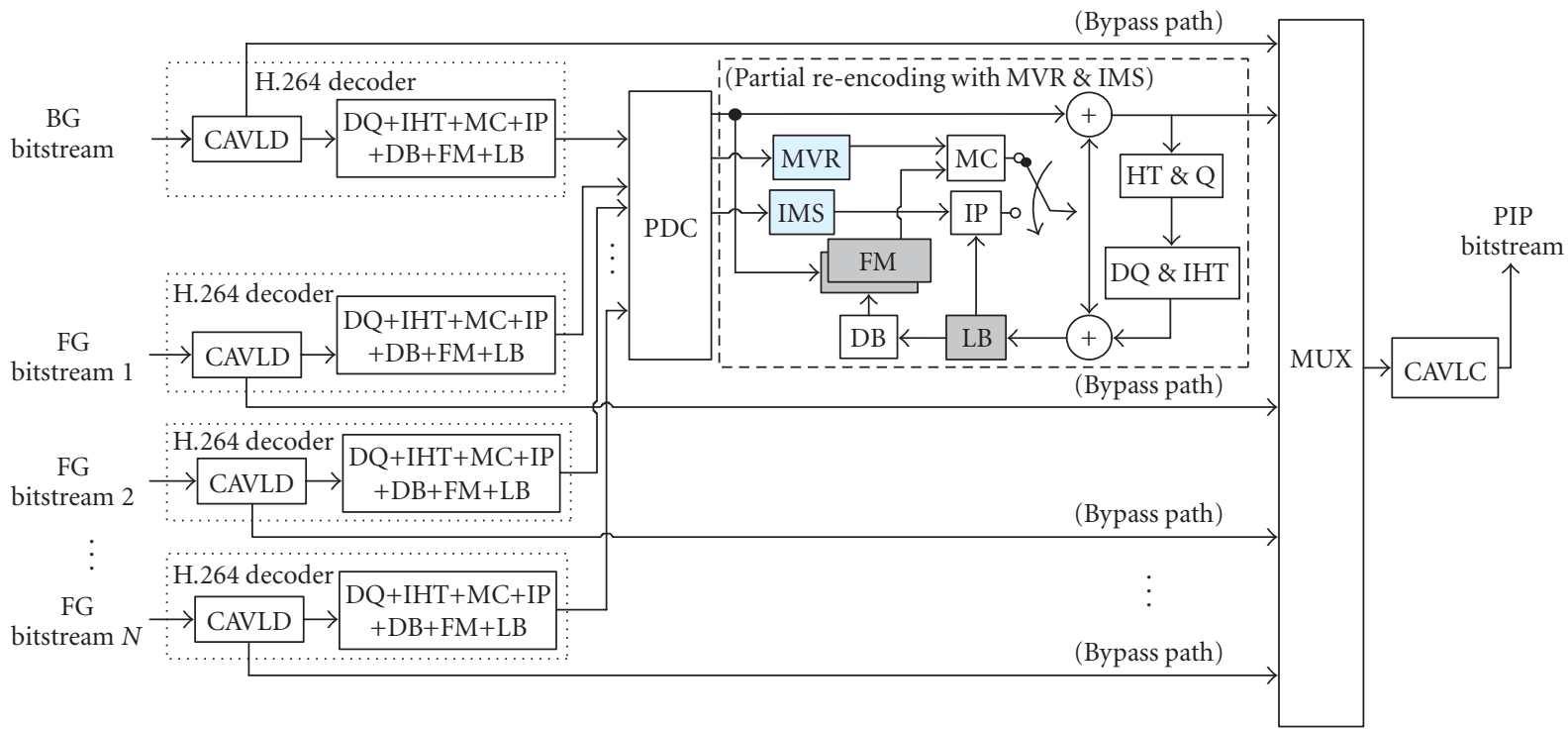

FIGURE 5: Intermediate architecture of RFMT with the MVR and the IMS refinement.

refine the decoded pixels of the affected blocks. In addition to one shared FM, the transcoding process is the same as that in Figure 5.

In case the PIP scenario generates the background block with top and left pixels next to the foreground pictures, our RFMT needs to decode each foreground bitstreams. Then, the transcoder switches the mode of this block to DC mode and computes the new residue according to the reconstructed values of two foreground pictures. Moreover, if the foreground pictures occupy the whole frame, the feature of channel preview is realized with the degenerated architecture of Figure 7. The remaining issues are how the IMS and the MVR modules deal with the wrong reference problem of back- ground bitstream. There are two goals: refining the affected blocks efficiently and deciding the minimal subset of refined block while retaining the visual quality of transcoded bitstream.

\subsubsection{Intramode switching}

For the intracoded $w$-MBs, we need to change the intramodes to fix the wrong reference problem since the intraprediction is performed in the spatial domain. The neighboring samples of the already encoded blocks are used as the prediction reference. Thus, when we replace parts of the background picture with the foreground pixels, the MBs 


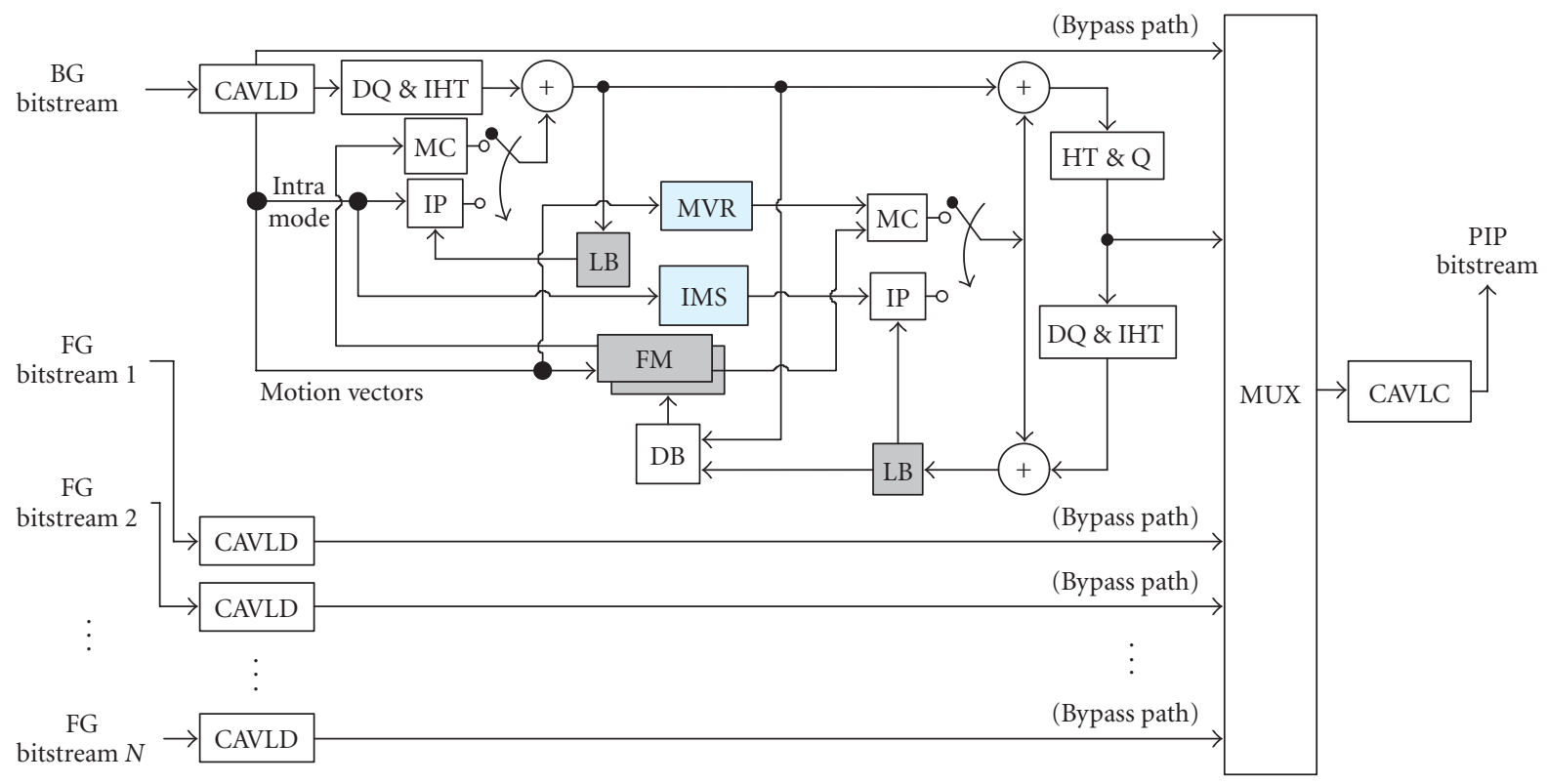

FIGURE 6: Final architecture of RFMT with shared frame memory for the constrained FG bitstreams.

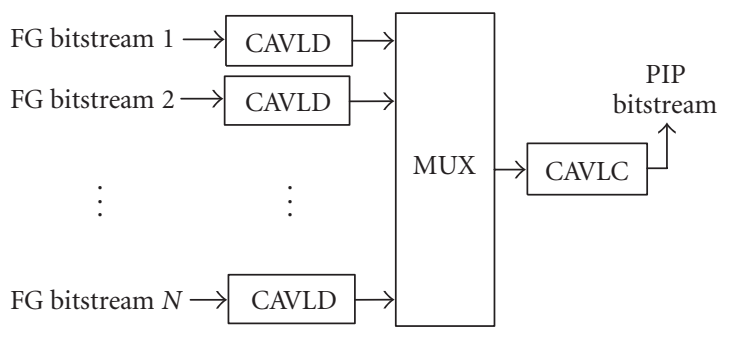

FIgURE 7: A transcoding scheme for channel preview.

around the borders may have visual artifacts due to the newly inserted samples. Without drift error correction, the distortion propagates spatially all over the whole frame via the intra prediction process in a raster scanning order. A straightforward refinement approach is to apply the R-D optimized (RDO) mode decision to find the best intra mode from the available pixels and then reencode new residue.

To reduce complexity we propose an intramode switching (IMS) technique for the intracoded $w$-MBs since the best reference pixels should come from the same region. The mode switching approach selects the best mode from the more probable intraprediction modes.

Each $4 \times 4$ block within a MB could be classified according to the intramodes as shown in Figure 8. Similarly, the mode of the $w$-block should be refined while the modes of $p$-blocks are unchanged. For the $w$-blocks, the IMS is performed according to the relative position with respect to the foreground pictures as shown in Figure 9. To speed up the IMS process, a table lookup method is used to select the new intramode according to the original intramode and the rel-

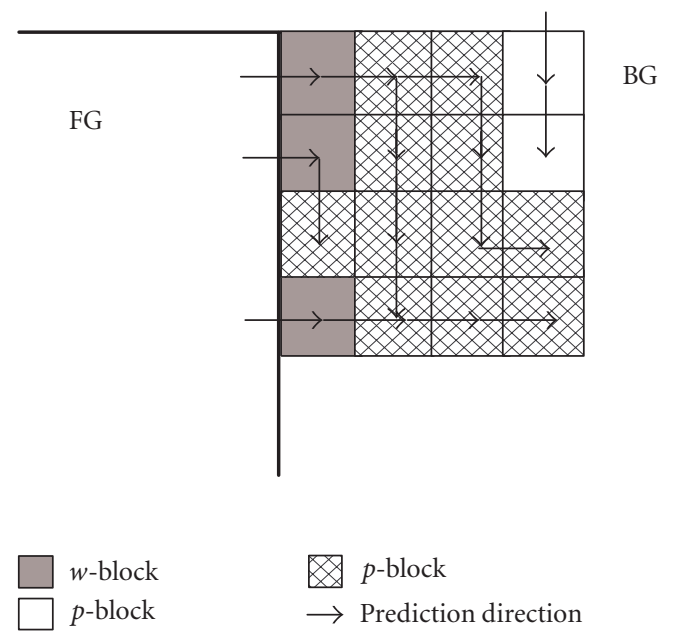

FIGURE 8: The wrong intrareference problem within a macroblock depending on the intramodes.

ative position. Tables 2 and 3 enumerate the IMS selection exhaustively.

With the refined intramode, we compute the new residue and coded block patterns. It should be noted that only the reconstructed quantized values are used as the original video is unavailable. Given that the $n$th $4 \times 4$ block is the $w$-block. The refinement of the $n$th $4 \times 4$ block is defined by

$$
r_{n}^{\prime}=\overline{x_{n}}-\operatorname{IP}_{2}\left(\overline{x_{j}}\right)=\overline{r_{n}}+\operatorname{IP}_{1}\left(\overline{x_{i}}\right)-\operatorname{IP}_{2}\left(\overline{x_{j}}\right),
$$

where the symbol $\overline{x_{n}}$ denotes the decoded pixel. The symbols $\operatorname{IP}_{1}\left(\overline{x_{i}}\right)$ and $\operatorname{IP}_{2}\left(\overline{x_{j}}\right)$ denote intraprediction from the reference pixels $\overline{x_{i}}$ and $\overline{x_{j}}$ by using the original mode, and the new mode respectively. The symbol $\overline{r_{n}}$ is the decoded 


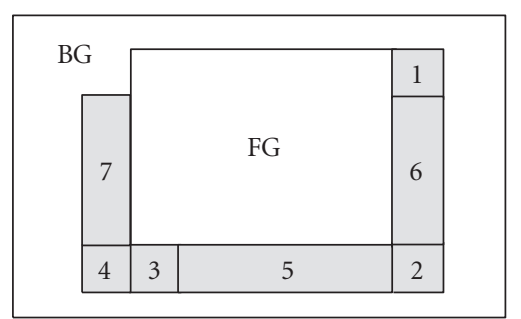

FIgURE 9: Relative position of each case in intramode switching method.

TABle 2: Cases of Intra4 mode switching.

\begin{tabular}{l|lcl}
\hline Case & Corresponding & Original Mode* & Switched Mode* \\
& $4 \times 4$ block & & 0 \\
\hline 1 & Left column of blocks & $1,2,4,5,6,8$ & 2 \\
2 & Top left of block & $4,5,6$ & 1 \\
3 & Top row of blocks & $0,2,3,4,5,6,7$ & 0 \\
4 & Top right of blocks & 3,7 & 1 \\
5 & Top row of blocks & $0,2,3,4,5,6,7$ & 0 \\
6 & Left column of blocks & $1,2,4,5,6,8$ & 0 \\
7 & Right column of blocks & 3,7 & \\
\hline
\end{tabular}

*0: Intra_ $4 \times 4$ _Vertical

1: Intra_ $4 \times 4$ _Horizontal

2: Intra_ $4 \times 4$ _DC

3: Intra_4 $\times 4$ _Diagonal_Down_Left

4: Intra_4 $\times 4$ _Diagonal_Down_Right

5: Intra_4 $\times 4$ _Vertical_Right

6: Intra_ $4 \times 4$ _Horizontal_Down

7: Intra_ $4 \times 4$ _Vertical_Left

8: Intra_4 $\times 4$ _Horizontal_Up

TABLE 3: Cases of Intra16 mode switching.

\begin{tabular}{l|cc}
\hline Case & Original Mode* & Switched Mode* \\
\hline 1,6 & $1,2,3$ & 1 \\
2 & 3 & 2 \\
3,5 & $0,2,3$ & 1 \\
\hline
\end{tabular}

*0: Intra_ $16 \times 16$ _Vertical

1: Intra_ $16 \times 16 \_$Horizontal

2: Intra_16 $\times 16 \_D C$

3: Intra_16 $\times 16 \_$Plane

residue extracted from the source bitstream. Then, the refined residue is requantized and dequantized as

$$
\begin{aligned}
\overline{r_{n}^{\prime}} & =P_{d} \cdot P_{e} \cdot r_{n}^{\prime}=P_{d} \cdot P_{e} \cdot\left[\overline{r_{n}}+\operatorname{IP}_{1}\left(\overline{x_{i}}\right)-\operatorname{IP}_{2}\left(\overline{x_{j}}\right)\right] \\
& =P_{d} \cdot P_{e} \cdot \overline{r_{n}}+P_{d} \cdot P_{e} \cdot \operatorname{IP}_{1}\left(\overline{x_{i}}\right)-P_{d} \cdot P_{e} \cdot \operatorname{IP}_{2}\left(\overline{x_{j}}\right) \\
& =\overline{r_{n}}+\operatorname{IP}_{1}\left(\overline{x_{i}}\right)+e_{i}-\operatorname{IP}_{2}\left(\overline{x_{j}}\right)-e_{j},
\end{aligned}
$$

where the symbols $e_{i}$ and $e_{j}$ are the quantization errors of $\mathrm{IP}_{1}\left(\overline{x_{i}}\right)$ and $\mathrm{IP}_{2}\left(\overline{x_{j}}\right)$. Lastly, the reconstructed data of the $n$th $4 \times 4$ block is shown in as

$$
\overline{x_{n}^{\prime}}=\overline{r_{n}^{\prime}}+\operatorname{IP}_{2}\left(\overline{x_{j}}\right)=\overline{r_{n}}+\operatorname{IP}_{1}\left(\overline{x_{i}}\right)+\left(e_{i}-e_{j}\right)=\overline{x_{n}}+e_{n},
$$

where the symbol $e_{n}$ denotes the refinement error due to the additional quantization process.

For the $p$-blocks, we recalculate the coefficients with the refined samples of $w$-blocks. The refinement of $w$-blocks may incur drift error that is amplified and propagated to the subsequent $p$-blocks by the intraprediction process. In order to alleviate the error propagation, we recalculate the coefficients of $p$-blocks based on the new reference samples with the original intramodes as shown in (6), where we assume the $m$ th $4 \times 4$ block is the intracoded $p$-block that uses the decoded data of the $n$th $4 \times 4$ block as prediction,

$$
\begin{aligned}
r_{m}^{\prime} & =\overline{x_{m}}-\operatorname{IP}_{1}\left(\overline{x_{n}^{\prime}}\right)=\overline{r_{m}}+\operatorname{IP}_{1}\left(\overline{x_{n}}\right)-\operatorname{IP}_{1}\left(\overline{x_{n}^{\prime}}\right) \\
& =\overline{r_{m}}+\operatorname{IP}_{1}\left(\overline{x_{n}}-\overline{x_{n}^{\prime}}\right)=\overline{r_{m}}+\operatorname{IP}_{1}\left(e_{n}\right) .
\end{aligned}
$$

Similarly, the refined residue should be requantized and dequantized as represented in (7) where the symbol $e_{m}$ denotes the drift error in the $m$ th $4 \times 4$ block and is identical to the quantization error of intraprediction of refinement error $e_{n}$ in the $n$th $4 \times 4$ block:

$$
\begin{aligned}
& \overline{x_{m}^{\prime}}=\overline{r_{m}^{\prime}}+\operatorname{IP}_{1}\left(\overline{x_{n}^{\prime}}\right)=P_{d} \cdot P_{e} \cdot \overline{r_{m}}+P_{d} \cdot P_{e} \cdot \operatorname{IP}_{1}\left(e_{n}\right) \\
&+\operatorname{IP}_{1}\left(\overline{x_{n}^{\prime}}\right)=\overline{r_{m}}+\operatorname{IP}_{1}\left(e_{n}\right)+e_{m}+\operatorname{IP}_{1}\left(\overline{x_{n}^{\prime}}\right) \\
&= \overline{x_{m}}-\operatorname{IP}_{1}\left(\overline{x_{n}}\right)+\operatorname{IP}_{1}\left(\overline{x_{n}^{\prime}}\right)+\operatorname{IP}_{1}\left(e_{n}\right)+e_{m} \\
&= \overline{x_{m}}+\operatorname{IP}_{1}\left(\overline{x_{n}^{\prime}}-\overline{x_{n}}+e_{n}\right)+e_{m}=\overline{x_{m}}+e_{m} .
\end{aligned}
$$

Similarly, the next $p$-block can be derived:

$$
\begin{aligned}
& \overline{x_{m+1}^{\prime}}=\overline{x_{m+1}}+e_{m+1}, \\
& e_{m+1}=P_{d} \cdot P_{e} \cdot e_{m}-e_{m}, \quad m=1,2,3, \ldots .
\end{aligned}
$$

The generalized projection theory says that consecutive projections onto two nonconvex sets will reach a trap point beyond which future projections do not change the results [40]. After several iterations of error correction, the drift error cannot be further compensated. Therefore, we only perform error correction to the $p$-blocks within intracoded $w$-MB rather than all the subsequent $p$-blocks. We observe that error correction for the $p$-blocks within intracoded $w$-MB improves the averaged R-D performance up to $1.5 \mathrm{~dB}$. However, error correction for the intracoded $p$-MBs has no significant quality improvement.

\subsubsection{Motion vector remapping}

The motion information of intercoded $w$-MBs needs to be reencoded since the motion vectors of the original bitstreams point to wrong reference samples after the embedding process, since only the motion vector difference is encoded instead of the full scale motion vector. Owing to such prediction dependency, the new foreground video creates the wrong reference problem.

To solve the wrong reference issue, reencoding the motion information is necessary for the surrounding MBs near the borders between foreground and background videos. In H.264/AVC, the motion vector difference is encoded according to the neighboring three motion vectors rather than the motion vector itself. Hence an identical motion vector predictor is needed for both encoder and decoder. However, due 
to foreground picture insertion, the motion compensation of background blocks may have wrong reference blocks from the new foreground pictures. Consequently, the incorrect motion vectors cause serious prediction error propagated to subsequent pictures through the motion compensation process.

Within the background pictures, the reference pixels pointed by the motor vector may be lost or changed. For the MBs with wrong prediction reference, the motion vectors need to be refined for correct reconstruction at the receiver. To provide good tradeoff between the R-D performance and complexity, only the MBs using the reference blocks across the picture borders are refined. The refinement process can be done with motion reestimation, mode decision, and entropy coding. It takes significant complexity to perform exhaustive motion reestimation and RDO mode decision for every MB with wrong prediction reference. Therefore, we use a motion vector remapping method (MVR) that has been extensively studied for MPEG-1/2/4 [20-22]. Before applying the MVR to the intercoded $w$-MBs, we select the Inter $4 \times 4$ mode as indicated in Figure 10. The MVR modifies the motor vector of every $4 \times 4 w$-block with a new motion vector pointing to the nearest of the four boundaries at the foreground picture. With the newly modified motion vectors, the prediction residue is recomputed and the HT transform is used to generate the new transform coefficients. Finally, the new motion vector and refined transform coefficients of $w$ blocks are entropy encoded as the final bitstream. The refinement process of MVR can be represented by (9), where the symbols $\operatorname{MC}\left(\overline{x_{i}}\right)$ and $\operatorname{MC}\left(\overline{x_{j}}\right)$ denote motion compensation from the reference pixels $\overline{x_{i}}$ and $\overline{x_{j}}$, respectively:

$$
\begin{aligned}
r_{n}^{\prime} & =\overline{x_{n}}-\operatorname{MC}\left(\overline{x_{j}}\right)=\overline{r_{n}}+\operatorname{MC}\left(\overline{x_{i}}\right)-\operatorname{MC}\left(\overline{x_{j}}\right) \\
& =\overline{r_{n}}+\operatorname{MC}\left(\overline{x_{i}}-\overline{x_{j}}\right) .
\end{aligned}
$$

The refined residue data is requantized and dequantized as

$$
\begin{aligned}
\overline{r_{n}^{\prime}} & =P_{d} \cdot P_{e} \cdot r_{n}^{\prime}=P_{d} \cdot P_{e} \cdot\left[\overline{r_{n}}+\operatorname{MC}\left(\overline{x_{i}}-\overline{x_{j}}\right)\right] \\
& =P_{d} \cdot P_{e} \cdot \overline{r_{n}}+P_{d} \cdot P_{e} \cdot \operatorname{MC}\left(\overline{x_{i}}-\overline{x_{j}}\right) \\
& =\overline{r_{n}}+\operatorname{MC}\left(\overline{x_{i}}-\overline{x_{j}}\right)+e_{n},
\end{aligned}
$$

where the symbol $e_{n}$ is the quantization error of $\operatorname{MC}\left(\overline{x_{i}}-\overline{x_{j}}\right)$. In the transcoded bitstream, the decoded signal of the $n$th $4 \times$ 4 block is represented in (11) where the symbol $e_{n}$ indicates the refinement error:

$$
\begin{aligned}
\overline{x_{n}^{\prime}} & =\overline{r_{n}^{\prime}}+\operatorname{MC}\left(\overline{x_{j}}\right) \\
& =\overline{r_{n}}+\operatorname{MC}\left(\overline{x_{i}}-\overline{x_{j}}\right)+e_{n}+\operatorname{MC}\left(\overline{x_{j}}\right)=\overline{x_{n}}+e_{n} .
\end{aligned}
$$

The refinement may occur at the border MBs with the skip mode. Since two neighboring motion vectors are used to infer the motion vector of an MB with the skip mode, the border MBs with the skip mode may be classified as two kinds of $w$-MBs due to the insertion of the foreground blocks. Firstly, for the $w$-MBs whose motion vectors that do not refer to a reference bock covered by the foreground pictures, the skip mode is changed to Inter $16 \times 16$ mode to compensate the mismatch of motion vectors by the motion inference. Secondly, for the $w$-MBs whose motion vectors point to reference blocks covered by the foreground pictures, the skip

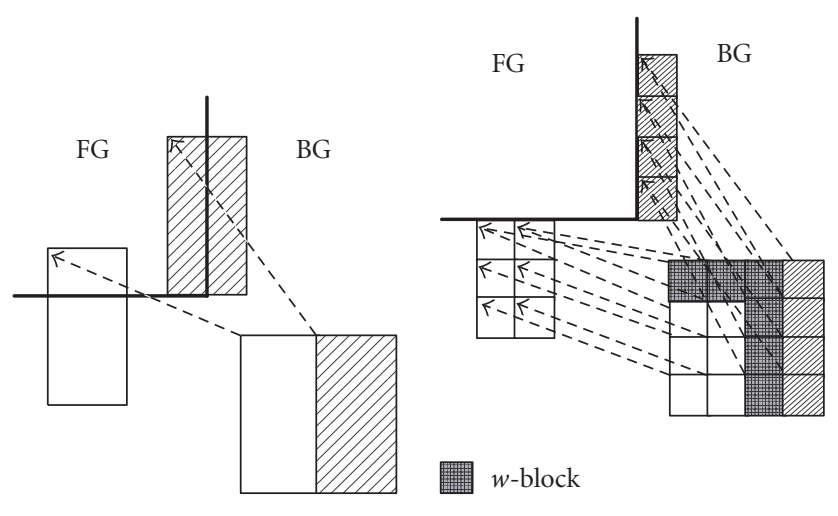

(a)

(b)

FIGURE 10: Illustration of motion vector remapping. (a) Original coding mode and motion vectors. (b) Using Inter $4 \times 4$ mode and refined motion vectors.

mode is changed to Inter $16 \times 16$ mode and the motion vector is refined to new position by the MVR method. Then, the refined coefficients are computed according to the new prediction.

To fix the wrong subpixel interpolation after inserting the foreground pictures, the blocks whose motion vectors point to the wrong subpixel positions are refined. H.264/AVC supports finer subpixel resolutions such as $1 / 2,1 / 4$, and $1 / 8$ pixel. The subpixel samples do not exist in the reference buffer for motion prediction. To generate the sub-pixel samples, a 6tap interpolation filter is applied to full-pixel samples for the subpixel location. The sub-pixel samples within 2-pixel range of picture boundaries are refined to avoid vertical and horizontal artifacts. The refinement is done by replacing the wrong subpixel motion vectors with the nearest full-pixel motion vectors and the new prediction residues are reencoded.

\subsection{Syntax level bypassing}

To minimize the transcoding complexity, the blocks within intercoded $p$-MBs and $n$-MBs are bypassed at the syntax level after the CAVLD. Since the blocks within $p$-MBs and $n$-MBs are not affected by the picture insertion directly, the syntax data can be forwarded unchanged to the multiplexer.

As for the intracoded frames, the affected blocks by video insertion are refined to compensate the drift error. We observe that the correction of $p$-blocks within the $w$-MBs can significantly improve the quality. In addition, the correction of intracoded $p$-MBs might get a bit of quality improvement with drastically increased complexity.

As for the intercoded frames, we examine the effectiveness of error compensation by (12). The $m$ th block is intercoded $p$-block and the residue is recomputed with the refined pixel values by

$$
\begin{aligned}
r_{m}^{\prime} & =\overline{x_{m}}-\operatorname{MC}\left(\overline{x_{i}^{\prime}}\right)=\overline{r_{m}}+\operatorname{MC}\left(\overline{x_{i}}\right)-\operatorname{MC}\left(\overline{x_{i}^{\prime}}\right) \\
& =\overline{r_{m}}+\operatorname{MC}\left(\overline{x_{i}}-\overline{x_{i}^{\prime}}\right) .
\end{aligned}
$$


TABLE 4: Corresponding operations of each block type during the VET transcoding.

\begin{tabular}{l|c|c}
\hline \multicolumn{2}{c|}{ Block type } & Operations \\
\hline \multirow{4}{*}{$w$-MB } & Intracoded $w$-block & IMS and CR \\
& Intercoded $w$-block & MVR and CR* \\
& Intracoded $p$-block & CR $^{*}$ \\
& Intercoded $p$-block & SLB \\
& $n$-block & SLB \\
& $p$-MB & SLB \\
& $n$-MB & SLB \\
\hline
\end{tabular}

*CR means coefficient recalculation.

TABle 5: Encoder parameters for the experiments.

\begin{tabular}{l|c}
\hline \multirow{2}{*}{ Frame size } & QCIF $(176 \times 144)$, \\
& CIF $(352 \times 288)$, \\
& SD $(720 \times 480)$, \\
Frame rate $(1920 \times 1088)$ \\
GOP structure & 30 frames $/ \mathrm{s}$ \\
Total frame & IPPPP..P \\
Intraperiod & 100 \\
Reference frame number & 15 \\
& 1 \\
Motion estimation range & 16 for QCIF, \\
& 32 for CIF, \\
& 64 for SD, \\
Quantization step size & and 176 for HD \\
\hline
\end{tabular}

Similarly, the transcoded data can be represented by (13) where the refinement error of the $w$-block is propagated to the next $p$-block:

$$
\begin{aligned}
\overline{x_{m}^{\prime}} & =\overline{r_{m}^{\prime}}+\operatorname{MC}\left(\overline{x_{i}^{\prime}}\right) \\
& =P_{d} \cdot P_{e} \cdot \overline{r_{m}}+P_{d} \cdot P_{e} \cdot \operatorname{MC}\left(\overline{x_{i}}-\overline{x_{i}^{\prime}}\right)+\operatorname{MC}\left(\overline{x_{i}^{\prime}}\right) \\
& =\overline{r_{m}}+\operatorname{MC}\left(\overline{x_{i}^{\prime}}\right)=\overline{x_{m}}-\operatorname{MC}\left(\overline{x_{i}}\right)+\operatorname{MC}\left(\overline{x_{i}^{\prime}}\right) \\
& =\overline{x_{m}}+\operatorname{MC}\left(\overline{x_{i}}-\overline{x_{i}^{\prime}}\right) .
\end{aligned}
$$

Let us assume the refinement of $w$-block performs well and the term of $\operatorname{MC}\left(\overline{x_{i}}-\overline{x_{i}^{\prime}}\right)$ is smaller than the quantization step size, it means that the quantization of $\operatorname{MC}\left(\overline{x_{i}}-\overline{x_{i}^{\prime}}\right)$ becomes zero. If our assumption is valid, the term $P_{d} \cdot P_{e} \cdot \operatorname{MC}\left(\overline{x_{i}}-\overline{x_{i}^{\prime}}\right)$ in (13) can be removed. Thus, the drift compensation of intercoded $p$-block has no quality improvement despite extra computations. In terms of complexity reduction, we bypass all the transform coefficients of $p-\mathrm{MB}$ and $n-\mathrm{MB}$ to the transcoded bitstream.

In summary, the proposed MW-VET deals with each type of block efficiently according to Table 4 . In addition, the partially reencoding method can preserve picture quality. For the applications requiring multigeneration transcoding, the deterioration caused by successive decoding and reencoding of the signals can be eliminated with the reuse of the coding information from the original bitstreams. As the motion

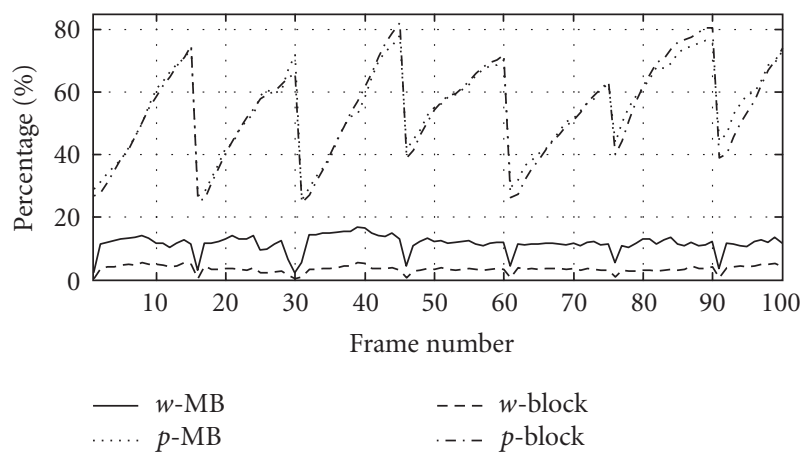

FIGURE 11: Percentage of the macroblock types and the block types during the VET transcoding.

compensation with multiple reference frames is applied, the proposed algorithm is still valid. Specifically, it first classifies the type of each block (i.e., $n$-block, $p$-block, and $w$-block according to Figure 3 ). The classification is based on whether the reference block is covered by foreground pictures and it does not matter what reference picture is chosen. In other words, the wrong reference problem with multiple reference frame feature is an extension of Figure 3. Then, the aforementioned MVR and SLB processes are applied to each type of intercoded block.

\section{EXPERIMENT RESULTS}

The R-D performance and execution time are compared based on the transcoding methods, test sequences, and picture insertion scenarios. For a fair comparison, all the transcoding methods have been implemented based on H.264/AVC reference software of version JM9.4. In addition, all the transcoders are built using Visual. NET compiler on a desktop with Windows XP, Intel P4 $3.2 \mathrm{GHz}$, and 2 Giga bytes DRAM. To further speed up the H.264/AVC based transcoding, the source code of the reference CAVLD module is optimized using a table lookup technique [41]. In the simulations, the test sequences are preencoded with the test conditions as shown in Table 5. The notation for each new transcoded bitstream is "background_foreground____y," where $x$ and $y$ are the coordinates of the foreground picture. The values of $x$ and $y$ need to be on the MB boundaries within the background picture. To evaluate the picture quality of each reconstructed sequence, the two original source sequences are combined to be the reference video source for peaksignal-to-noise ratio (PSNR) computation.

The percentage of each MB type and each $4 \times 4$ block type is shown in Figure 11. In general, the $p$-MBs occupy $30 \%$ to $80 \%$ of MBs and the percentage of the $w$-MBs is less than $15 \%$. In addition, the $w$-blocks occupy only $5 \%$ of the $4 \times 4$ blocks. Bypassing all the $p$-blocks that are $95 \%$ of blocks accelerates the transcoding process as shown in Table 6. On the average, as compared to the CPDT, the MW-VET can achieve 25 times of speedup with improved picture quality.

Table 7 lists the PSNR comparison to show the effectiveness of error correction for different kinds of blocks. The 
TABLE 6: Improvement of execution time and quality as compared to $\mathrm{CPDT}^{(1)}$

\begin{tabular}{l|l|cc}
\hline \multicolumn{2}{c|}{ VET combination } & \multirow{2}{*}{$\begin{array}{c}\text { Speed-up } \\
\text { ratio }\end{array}$} & $\begin{array}{l}\text { PSNR gain of } \\
\text { Luma component }\end{array}$ \\
\hline BG ${ }^{(2)}$ & FG $^{(3)}$ \& location & 25 & $+1.72 \mathrm{~dB}$ \\
Stefan & Mobile_1_1 & 28 & $+1.56 \mathrm{~dB}$ \\
Table & Carphone_1_1 & & \\
& Mobile_1_1 & \multirow{2}{*}{28} & $+1.18 \mathrm{~dB}$ \\
Stefan & Foreman_33_1 & & \\
& News_1_20 & & \\
& Coastguard_33_20 & & $+1.15 \mathrm{~dB}$ \\
& M\&D_1_1 & & \\
\cline { 2 - 2 } Table & Stefan_33_1 & 25 & \\
& Carphone_1_20 & & \\
& News_33_20 & & \\
\hline
\end{tabular}

(1) Intel P4 3.2 G, 2 GB SDRAM, Windows XP, and Visual. NET compiler.

(2) All are in SD resolution.

(3) All are in QCIF resolution.

TABLe 7: Effectiveness of error correction for different kinds of $p$ blocks.

\begin{tabular}{l|c}
\hline Methods & PSNR $(\mathrm{dB})$ \\
\hline Golden & 43.73 \\
CPDT & 42.02 \\
RFMT w/o EC & 41.18 \\
RFMT with EC for the $p$-blocks & 43.16 \\
in intra-coded $w$-MBs & 43.33 \\
RFMT with EC for all intracoded $p$-blocks & 43.14 \\
RFMT with EC for all intercoded $p$-blocks & \\
\hline
\end{tabular}

golden method is not a transcoding scheme. The R-D curves of golden method are obtained from encoding the original picture-in-picture source sequences. The inclusion of the R$\mathrm{D}$ curves of golden method is to highlight the upper bound of a transcoder. The error correction of $p$-blocks in the intracoded $w$-MBs can obtain a significant gain in picture quality. However, the error correction for other $p$-blocks almost has no quality improvement while the complexity increases dramatically. Therefore, the results verify our derivations in Section 4.

The R-D performance of different approaches at various bit rates and different VET scenarios are compared. We embedded one foreground picture into one background picture at different positions in Figures 12 and 13. The performance of RFMT is better than that of CPDT. At medium and high bit rates, the RFMT can offer up to $1.5 \mathrm{~dB}$ improvement in PSNR. Even through the mode and motion vectors obtained by our IMS and MVR is not always the optimal solution, the simulation results show that our IMS and MVR approaches provide a solution close to the optimal case. In the comparison, we have plotted the R-D curves named as RFMT_RDO to show the optimal R-D performance when the partial reen-

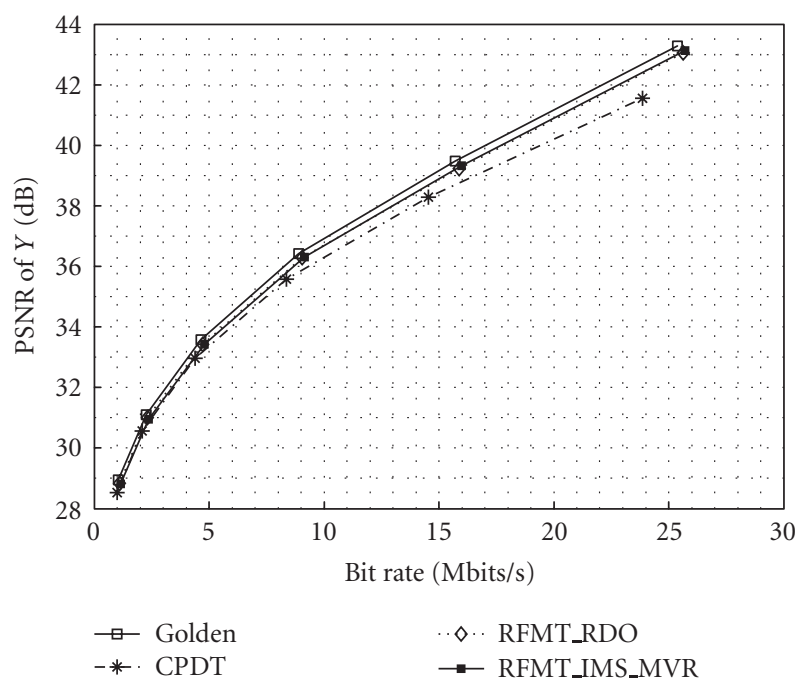

(a) Table_SD_Carphone_QCIF _1_1

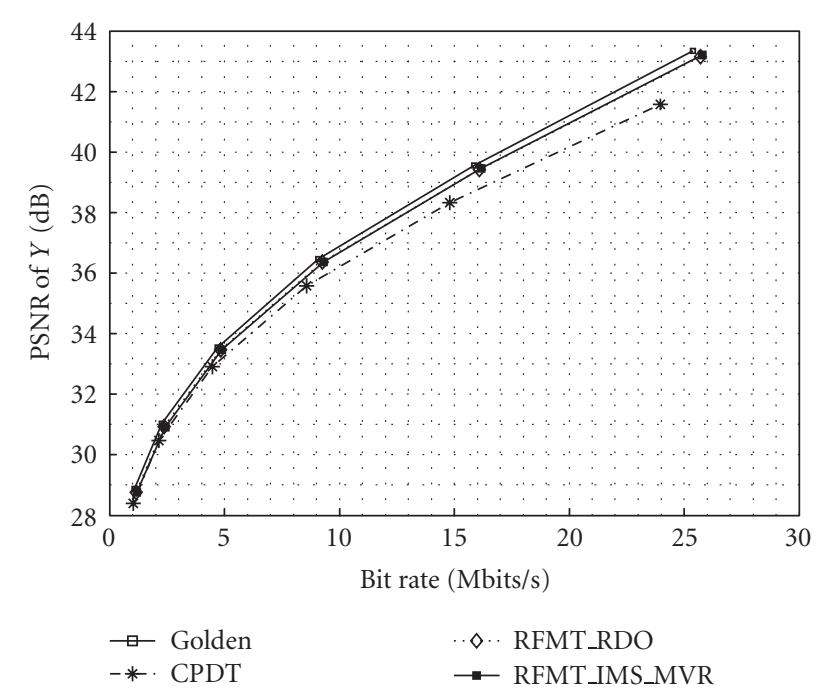

(b) Table_SD_Carphone_QCIF_33_20

FIGURE 12: Rate-distortion performance of the luminance component by one foreground embedding for Table_SD_Carphone_QCIF.

coding is performed under RDO mode decision and motion vector reestimation. It could be observed that the R-D performance of RFMT with IMS and MVR is very close to that of RFMT_RDO.

Figure 14 shows the R-D curve of transcoding bitstreams that embed four foreground pictures onto one background picture at the same time. As compared with the oneforeground VET scenarios, the performance has a little degradation because that the ratio of $w$-blocks and $p$-blocks increases. Figure 15 shows the performance of multigeneration transcoding that embeds one foreground picture to the background picture every generation. Our MW-VET can retain the R-D performance while the CPDT degrades every generation. Thus, the proposed MW-VET is robust for the multigeneration transcoding. 


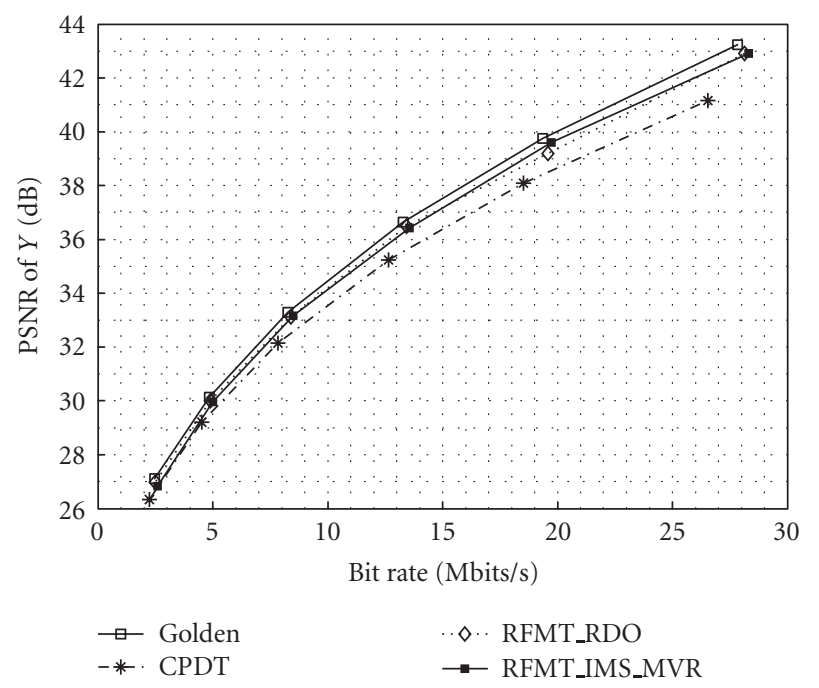

(a) Mobile_SD_Foreman_QCIF_1_1

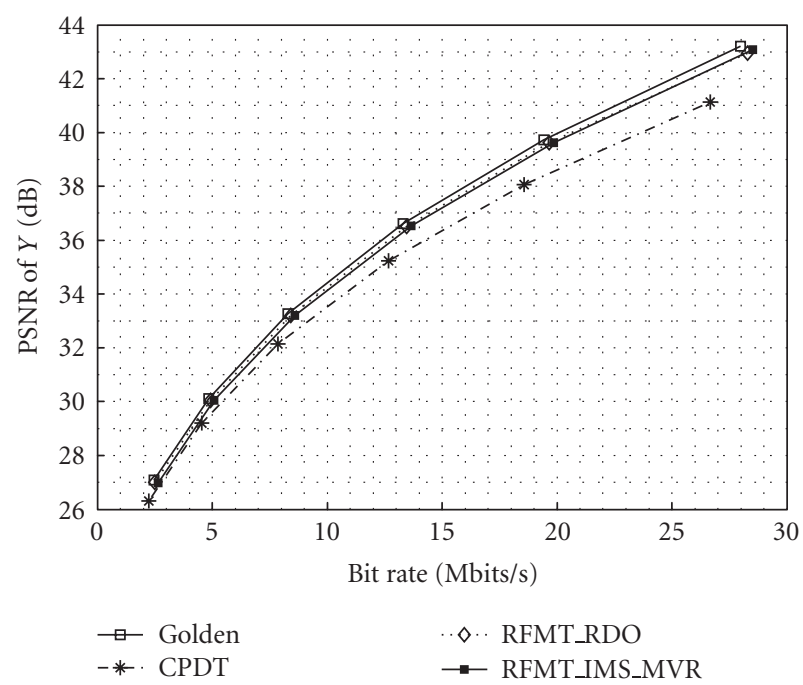

(b) Mobile_SD_Foreman_QCIF_33_20

FIGURE 13: Rate-distortion performance of the luminance component by one foreground embedding for Mobile_SD_Foreman_QCIF.

\section{CONCLUSIONS}

In this paper, we present an efficient multiple-window video embedding transcoder (MW-VET) to embed the multiple foreground videos into one background video. The pictures are inserted at the MB-aligned positions to retain high flexibility. To minimize complexity with negligible quality loss, the MW-VET uses three novel approaches including slice group-based transcoding (SGT), reduced frame memory transcoding (RFMT), and syntax level bypassing (SLB). These approaches are used based on the H.264/AVC coding standard compliant prediction schemes.

As the prediction is applied to the slice-aligned data partitions within the original bitstreams, the SGT parses and

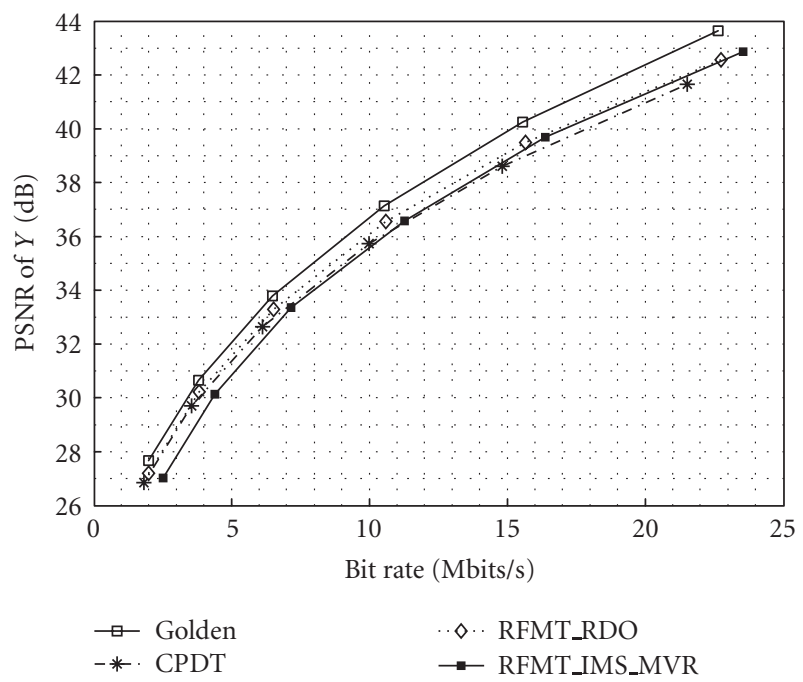

(a) Mobile_SD_M\&D_QCIF_1_1_Stefan_QCIF_ 33_1_Carphone_ QCIF_1_20_News_QCIF_33_20

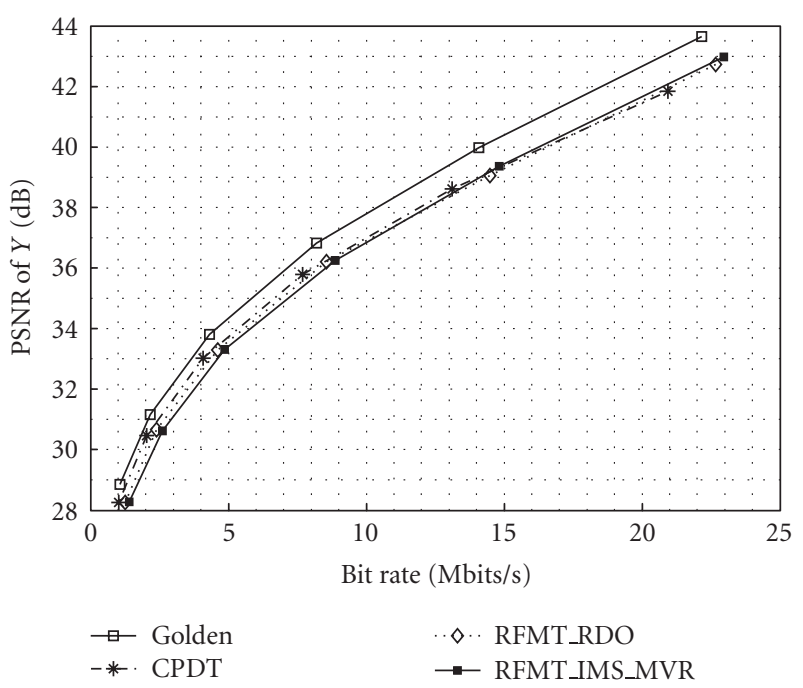

(b) Table_SD_M\&D_QCIF_1_1_Stefan_QCIF_33_1_Carphone_QCIF_ 1_20_News_QCIF_33_20

FIGURE 14: Rate-distortion performance of the luminance component by four foregrounds embedding.

merges the bitstreams directly. When the prediction is applied to the region-aligned data partitions, the MBs with wrong prediction reference are processed with the RFMT that partially reencodes the blocks to minimize the number of refined blocks. To handle intercoded and intracoded blocks that suffer from the wrong reference problem, the RFMT employs motion vector remapping (MVR), and intramode switching (IMS), respectively. The unaffected MBs are handled by the SLB in terms of transcoding throughput and picture quality.

Our results show that the MW-VET as compared to the cascaded pixel domain transcoder (CPDT) can significantly reduce the processing complexity by 25 times with similar or higher R-D performance. In addition, the MW-VET can 


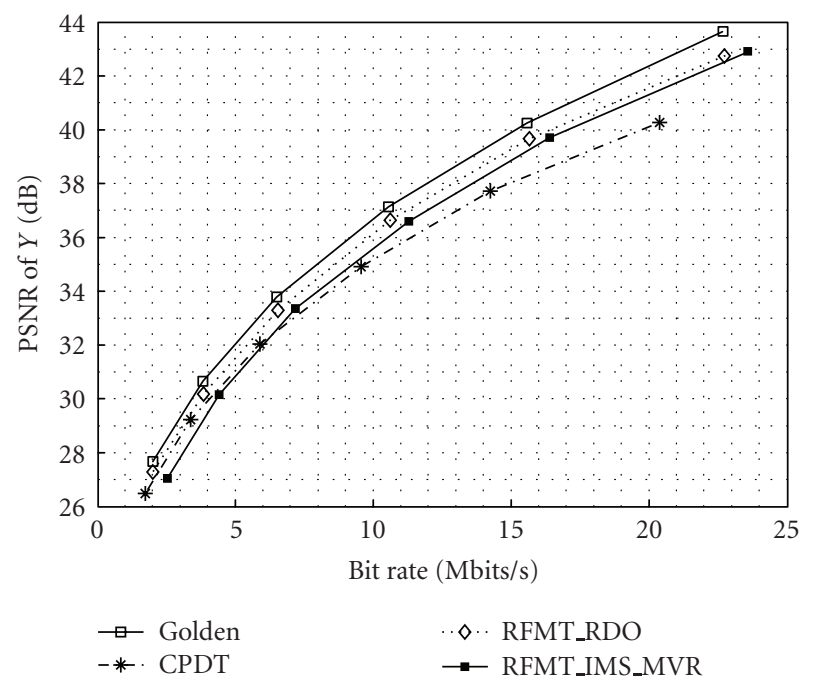

(a) Mobile_SD_M\&D_QCIF_1_1_Stefan_QCIF_33_1_Carphone_QCIF_ 1_20_News_QCIF_33_20

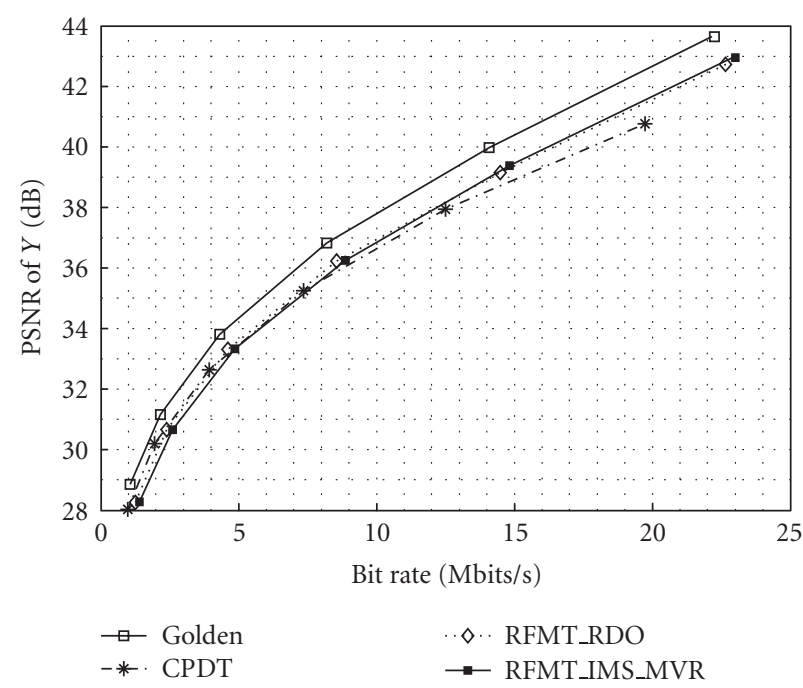

(b) Table_SD_M\&D_QCIF_1_1_Stefan_QCIF_33_1_Carphone_QCIF_ 1_20_News_QCIF_33_20

FIGURE 15: Rate-distortion performance of the luminance component by four foregrounds embedding and multi-generation transcoding.

achieve up to $1.5 \mathrm{~dB}$ quality improvement in PSNR. Based on the MW-VET, the quality improvement over the CPDT is significant for multigeneration transcoding.

\section{APPENDICES}

\section{A. WHY TRANSFORM DOMAIN APPROACHES ARE INEFFICIENT FOR H.264/AVC-BASED TRANSCODING}

In this appendix, we will show that it is inefficient to develop a transcoder in the transform domain as commonly proposed for previous standards such as MPEG-1/2/4. There are several reasons to support such a claim.

(1) In H.264/AVC, the transformation and quantization processes are so optimized that traverse back to the pixel domain is not as expensive as before.

(2) The intraprediction and deblocking filter introduce stronger spatial domain error propagation although they are effective to exploit the spatial redundancy.

(3) The IMC becomes inefficient when the motion compensation uses quarter-pixel resolution combined with 6-tap interpolation.

The following text will describe the detailed study to support such a claim.

\section{A.1. Integer transform with quantization scaling}

The transformation used in H.264/AVC is an integrated transform with quantization scaling, which means the scaling multiplication is merged with the quantization. The integer transform with quantization scaling is performed with simple integer operations such as shifting and addition, which indicates no rounding mismatch between the encoder and the decoder. The relationship between the pixel values at the encoder and the decoder can be represented by

$$
\operatorname{IHT}\{\mathrm{DQ}\{\mathrm{Q}[\mathrm{HT}(x)]\}\}=\bar{x},
$$

where $x$ and $\bar{x}$ mean the original data and the decoded data respectively. However, the data after de-quantization is not the original HT coefficients:

$$
\mathrm{DQ}\{\mathrm{Q}[\mathrm{HT}(x)]\} \neq \mathrm{HT}(x) \text {. }
$$

In order to obtain the transform coefficients at the transcoder side, an inverse operation of quantization is needed. The inverse quantization of HT coefficients is derived as follows. The following shows the quantization of HT coefficients $Y_{i, j}$ :

$$
\begin{gathered}
Z_{i, j}=\left(Y_{i, j} \times \mathrm{MF}_{i, j}+f\right) \gg S_{1}, \\
\text { with } \quad S_{1}=15+\left\lfloor\frac{\mathrm{QP}}{6}\right\rfloor, \quad f=\frac{2^{S_{1}}}{3} \quad \text { or } \quad \frac{2^{S_{1}}}{6} .
\end{gathered}
$$

The following shows that the data after the dequantization is different from the original HT coefficients:

$$
\begin{aligned}
W_{i, j} & =\left(Z_{i, j} \times V_{i, j}\right) \ll S_{2} \\
& =\left\{\left[\left(Y_{i, j} \bullet \mathrm{MF}_{i, j}+f\right) \gg S_{1}\right] \bullet V_{i, j}\right\} \ll S_{2} \neq Y_{i, j}
\end{aligned}
$$$$
\text { with } S_{2}=\left\lfloor\frac{\mathrm{QP}}{6}\right\rfloor \text {. }
$$

The symbols $\mathrm{MF}_{i, j}$ and $V_{i, j}$ are multiplication and rescaling factors, respectively, as defined in H.264/AVC standard. To obtain the HT coefficients, the dequantization process should be replaced by converting quantized coefficients to dequantized HT coefficients. The process is computed as

$$
Y_{i, j}^{\prime}=\frac{\left(Y_{i, j} \ll S_{1}\right)}{\mathrm{MF}_{i \cdot j}} .
$$

However, (A.5) requires a division operation with higher complexity and additional rounding error. 


\section{A.2. Directional intra prediction}

The intraprediction as defined in the spatial domain poses challenges to the transform domain transcoding. To implement intraprediction in the transform domain significantly increases complexity because the HT transform is not orthogonal, which means the transpose is not equal to the inverse for HT transform as represented in below:

$$
\begin{aligned}
\mathrm{HT}\left(\left[\begin{array}{cccc}
\times & \times & \times & \times \\
\times & \times & \times & \times \\
\times & \times & \times & \times \\
A & B & C & D
\end{array}\right]\right) & =\left(C_{f}\left[\begin{array}{cccc}
\times & \times & \times & \times \\
\times & \times & \times & \times \\
\times & \times & \times & \times \\
A & B & C & D
\end{array}\right] C_{f}^{T}\right) \\
& \neq\left(C_{f}\left[\begin{array}{cccc}
\times & \times & \times & \times \\
\times & \times & \times & \times \\
\times & \times & \times & \times \\
A & B & C & D
\end{array}\right] C_{f}^{-1}\right) .
\end{aligned}
$$

The detailed operations of HT domain intraprediction could be found in [42]. As compared to the pixel domain intraprediction, the computation increases especially in the number of multiplication as listed in Table 8.

\section{A.3. In-the-loop deblocking filtering}

The de-blocking filter defined in the spatial domain introduces mismatch error during HT domain transcoding. Particularly, the deblocked pixels stored in the reference frame memory are used for motion compensation. Thus, mismatch error will propagate to the next frames via motion compensation until the subsequent intracoded frame or slices at the decoder. To prevent the mismatch error, implementing the de-blocking filter in the HT domain is required. However, this kind of implementation increases the complexity and memory requirement.

\section{A.4. Subpixel interpolation}

The complexity of HT domain IMC increases due to the 6tap interpolator defined in H.264/AVC. Detailed derivations are given in the following. A $4 \times 4$ motion-compensated block can be represented as the summation of four blocks in the spatial domain where

$$
\begin{gathered}
B_{\text {pred(4×4)_full_pel }}=\sum_{k=1}^{4} V_{k(4 \times 4)} B_{k} H_{k(4 \times 4),},\left[\begin{array}{cc}
0 & I_{h} \\
0 & 0
\end{array}\right], \\
V_{1(4 \times 4)}=V_{2(4 \times 4)} \\
H_{1(4 \times 4)}=H_{3(4 \times 4)}=\left[\begin{array}{cc}
0 & 0 \\
I_{w} & 0
\end{array}\right], \\
V_{3(4 \times 4)}=V_{4(4 \times 4)}=\left[\begin{array}{cc}
0 & 0 \\
I_{4-h} & 0
\end{array}\right], \\
H_{2(4 \times 4)}=H_{4(4 \times 4)}=\left[\begin{array}{cc}
0 & I_{4-w} \\
0 & 0
\end{array}\right] .
\end{gathered}
$$

TABLE 8: Computation complexity of intraprediction for different approaches.

\begin{tabular}{c|cc|cc}
\hline \multirow{2}{*}{ Mode* $^{*}$} & \multicolumn{2}{|c|}{ HT domain } & \multicolumn{2}{c}{ Spatial domain } \\
& Mul** & Addition & Mul $^{* *}$ & Addition \\
\hline 0 & 8 & 12 & 0 & 128 \\
1 & 8 & 12 & 0 & 128 \\
2 & 1 & 1 & 0 & 135 \\
3 & 168 & 136 & 0 & 159 \\
4 & 232 & 200 & 0 & 160 \\
5 & 192 & 176 & 0 & 155 \\
6 & 192 & 176 & 0 & 155 \\
7 & 128 & 112 & 0 & 152 \\
8 & 64 & 48 & 0 & 155 \\
\hline
\end{tabular}

$* 0$ Intra_ $4 \times 4$ _Vertical

$* 1$ Intra_ $4 \times 4$ _Horizontal

*2 Intra_4 $\times 4$ _DC

*3 Intra_4 $\times 4$ _Diagonal_Down_Left

*4 Intra_4 $\times 4$ 4_Diagonal_Down_Right

$* 5$ Intra_ $4 \times 4$ 4_Vertical_Right

*6 Intra_4 $\times 4$ 4_Horizontal_Down

*7 Intra_4 $\times 4$ __Vertical_Left

$* 8$ Intra_ $4 \times 4$ _Horizontal_Up

$* *$ : Multiplication

We start the discussion of IMC from a $4 \times 4$ block with integer MV. The HT coefficients of prediction block can be calculated from four HT blocks as indicated by

$$
\begin{aligned}
& \operatorname{HT}\left(B_{\text {pred(4×4)_full_pel }}\right) \\
&= \operatorname{HT}\left(\sum_{k=1}^{4} V_{k(4 \times 4)} B_{k} H_{k(4 \times 4)}\right) \\
&= \sum_{i=1}^{4} \operatorname{HT}\left(V_{k(4 \times 4)} B_{k} H_{k(4 \times 4)}\right) \\
&= \sum_{k=1}^{4}\left(C_{f} V_{k(4 \times 4)} C_{f}^{-1}\right)\left(C_{f} B_{k} C_{f}^{-1}\right) \\
& \times\left(C_{f} H_{k(4 \times 4)} C_{f}^{T}\right)\left(C_{f} V_{k(4 \times 4)} C_{f}^{-1}\right) \\
&= \sum_{k=1}^{4} \operatorname{HT}\left(B_{k}\right)\left[\begin{array}{llll}
c & 0 & 0 & 0 \\
0 & a & 0 & 0 \\
0 & 0 & c & 0 \\
0 & 0 & 0 & a
\end{array}\right]\left(C_{f} H_{k(4 \times 4)} C_{f}^{T}\right) .
\end{aligned}
$$

The terms of $\left(C_{f} V_{i(4 \times 4)} C_{f}^{-1}\right)$ and $\left(C_{f} H_{i(4 \times 4)} C_{f}^{T}\right)$ can be precomputed and stored in memory. The computation of (A.8) needs 576 multiplications and 384 additions.

The subpixel interpolation filter increases the complexity of transform domain IMC. The half-pixel sample is interpolated from integral pixel samples by applying a 6-tap finite impulse response (FIR) filter, whose weights are $(1,-20,20$, $5 / 8,-5,1) / 32$. The HT coefficients of a prediction block on the half-pixel position have to be calculated from nine blocks 
as indicated in the following equation:

$$
\begin{aligned}
& \mathrm{HT}\left(B_{\text {pred(4×4)_sub_pel }}\right) \\
& =\sum_{k=1}^{9}\left[\begin{array}{c}
\left(C_{f} V_{\mathrm{avg}(4 \times 9)} V_{k(9 \times 4)}^{\prime} C_{f}^{-1}\right) \\
\operatorname{HT}\left(B_{k}\right)\left[\begin{array}{cccc}
c & 0 & 0 & 0 \\
0 & a & 0 & 0 \\
0 & 0 & c & 0 \\
0 & 0 & 0 & a
\end{array}\right] \\
\left(C_{f} H_{k(4 \times 9)}^{\prime} H_{\mathrm{avg}(9 \times 4)} C_{f}^{T}\right)
\end{array}\right],
\end{aligned}
$$

where

$$
\begin{aligned}
& V_{1(9 \times 4)}^{\prime}=V_{2(9 \times 4)}^{\prime}=V_{3(9 \times 4)}^{\prime}=\left[\begin{array}{cc}
0 & I_{h} \\
0 & 0
\end{array}\right], \\
& V_{1(9 \times 4)}^{\prime}=V_{2(9 \times 4)}^{\prime}=V_{3(9 \times 4)}^{\prime}=\left[\begin{array}{cc}
0 & I_{h} \\
0 & 0
\end{array}\right] \text {, } \\
& H_{1(4 \times 9)}^{\prime}=H_{2(4 \times 9)}^{\prime}=H_{3(4 \times 9)}^{\prime}=\left[\begin{array}{cc}
0 & 0 \\
I_{w} & 0
\end{array}\right] \text {, } \\
& V_{4(9 \times 4)}^{\prime}=V_{5(9 \times 4)}^{\prime}=V_{6(9 \times 4)}^{\prime}=\left[\begin{array}{c}
0 \\
I_{4} \\
0
\end{array}\right] \text {, } \\
& H_{4(4 \times 9)}^{\prime}=H_{5(4 \times 9)}^{\prime}=H_{6(4 \times 9)}^{\prime}=\left[\begin{array}{lll}
0 & I_{4} & 0
\end{array}\right] \text {, } \\
& V_{7(9 \times 4)}^{\prime}=V_{8(9 \times 4)}^{\prime}=V_{9(9 \times 4)}^{\prime}=\left[\begin{array}{cc}
0 & 0 \\
I_{5-h} & 0
\end{array}\right] \text {, } \\
& H_{7(4 \times 9)}^{\prime}=H_{8(4 \times 9)}^{\prime}=H_{9(4 \times 9)}^{\prime}=\left[\begin{array}{cc}
0 & I_{5-w} \\
0 & 0
\end{array}\right] \text {, } \\
& V_{\mathrm{avg}(4 \times 9)}=\frac{1}{32}\left[\begin{array}{ccccccccc}
1 & -5 & 20 & 20 & -5 & 1 & 0 & 0 & 0 \\
0 & 1 & -5 & 20 & 20 & -5 & 1 & 0 & 0 \\
0 & 0 & 1 & -5 & 20 & 20 & -5 & 1 & 0 \\
0 & 0 & 0 & 1 & -5 & 20 & 20 & -5 & 1
\end{array}\right] \text {, }
\end{aligned}
$$

$H_{\text {avg }(4 \times 9)}$ is the transpose of $V_{\text {avg }(4 \times 9)}$.

The computation of (A.9) requires 1296 multiplications and 864 additions. Assume that the frame size is $M$ by $N$ and the probability of full-pixel MV is $\alpha$, the total amount of computation for the HT domain IMC involves with

$$
\begin{aligned}
576 \times & \frac{M N}{4} \times \alpha+1296 \times \frac{M N}{4} \times(1-\alpha) \\
& =\frac{M N}{4}(1296-720 \alpha)
\end{aligned}
$$

multiplications and

$384 \times \frac{M N}{4} \times \alpha+864 \times \frac{M N}{4} \times(1-\alpha)=\frac{M N}{4}(864-480 \alpha)$ additions.
As for the spatial domain IMC, the total amount of computation covers

$$
\begin{aligned}
& {[M(N-1)+N(M-1)+(M-1)(N-1)] \times 4} \\
& \quad=12 M N-8(M+N)+4
\end{aligned}
$$

multiplications and

$$
\begin{gathered}
{[M(N-1)+N(M-1)+(M-1)(N-1)] \times 5+64} \\
\times \frac{M N}{4} \times 2=47 M N-10(M+N)+5
\end{gathered}
$$

additions.

On the average, the SD resolution bitstream has $25 \%$ of motion vectors pointing to full-pixel locations and $75 \%$ of motion vectors pointing to half-pixel locations. Therefore, the complexity increase by subpixel interpolation in the HT domain is not preferable for transcoding. From the results of (A.11) and (A.12), the required multiplications and additions of HT domain IMC are about 23 times and 3 times as compared to that of spatial domain IMC, respectively.

\section{ACKNOWLEDGMENT}

This work was supported in part by the National Science Council of the Republic of China, under Grant NSC 95-2221E009-074-MY3.

\section{REFERENCES}

[1] ITU-T Rec. H.264 and ISO/IEC 14496-10 (MPEG4-AVC), "Advanced Video Coding for Generic Audiovisual Services," v1, May 2003; v2, January 2004; v3, September 2004; v4, July 2005.

[2] S. Wenger, "H.264/AVC over IP," IEEE Transactions on Circuits and Systems for Video Technology, vol. 13, no. 7, pp. 645-656, 2003.

[3] M. D. Nava and C. Del-Toso, "A short overview of the VDSL system requirements," IEEE Communications Magazine, vol. 40, no. 12, pp. 82-90, 2002.

[4] S. Naimpally, L. Johnson, T. Darby, R. Meyer, L. Phillips, and J. Vantrease, "Integrated digital IDTV receiver with features," IEEE Transactions on Consumer Electronics, vol. 34, no. 3, pp. 410-419, 1988.

[5] D. Gillies, R. Schweer, and H. Zibold, "VLSI realisations for picture in picture and flicker free television display," IEEE Transactions on Consumer Electronics, vol. 34, no. 1, pp. 253$261,1988$.

[6] M. Burkert, F. Frieling, U. Langenkamp, U. Libal, M. Mende, and G. Scheffler, "IC set for a picture-in-picture system with on-chip memory," IEEE Transactions on Consumer Electronics, vol. 36, no. 1, pp. 23-31, 1990.

[7] C. A. Mancini and C. P. Markhauser, "Microprocessor controlled picture in picture system," IEEE Transactions on Consumer Electronics, vol. 36, no. 3, pp. 375-379, 1990.

[8] M. Honzawa, M. Koyama, T. Hibino, H. Miyashita, and Y. Shiine, "New picture in picture LSI enhanced functionality for high picture quality," IEEE Transactions on Consumer Electronics, vol. 36, no. 3, pp. 387-394, 1990.

[9] L. D. Johnson, J. N. Pratt, and D. C. Greene, "Low cost picturein-picture for color TV receivers," IEEE Transactions on Consumer Electronics, vol. 36, no. 3, pp. 380-386, 1990. 
[10] S. Tsuchida and C. Yoshida, "Multi-picture system for high resolution wide aspect ratio screen," IEEE Transactions on Consumer Electronics, vol. 37, no. 3, pp. 313-319, 1991.

[11] G. W. Perkins, R. C. Hathaway, S. W. Lai, et al., "A low cost, monolithic, color picture-in-picture device," IEEE Transactions on Consumer Electronics, vol. 40, no. 3, pp. 306-316, 1994.

[12] A. Rick, T. Herfet, and S. J. Prange, "Digital color decoder for PIP-applications," IEEE Transactions on Consumer Electronics, vol. 42, no. 3, pp. 716-720, 1996.

[13] M. Brett and D. Wendel, "High performance picture-inpicture (PIP) IC using embedded DRAM technology," IEEE Transactions on Consumer Electronics, vol. 45, no. 3, pp. 698705, 1999.

[14] M. Schu, G. Scheffler, C. Tuschen, and A. Stolze, "System on silicon-IC for motion compensated scan rate conversion, picture-in-picture processing, split screen applications and display processing," IEEE Transactions on Consumer Electronics, vol. 45, no. 3, pp. 842-850, 1999.

[15] M. Schu, D. Wendel, C. Tuschen, M. Hahn, and U. Langenkamp, "System-on-silicon solution for high quality consumer video processing - the next generation," IEEE Transactions on Consumer Electronics, vol. 47, no. 3, pp. 412-419, 2001.

[16] C. Hentschel, R. J. Bril, Y. Chen, R. Braspenning, and T.H. Lan, "Video quality-of-service for consumer terminals-a novel system for programmable components," IEEE Transactions on Consumer Electronics, vol. 49, no. 4, pp. 1367-1377, 2003.

[17] I. Ahmad, X. Wei, Y. Sun, and Y.-Q. Zhang, "Video transcoding: an overview of various techniques and research issues," IEEE Transactions on Multimedia, vol. 7, no. 5, pp. 793-804, 2005.

[18] S.-F. Chang and D. G. Messerschmitt, "Compositing motioncompensated video within the network," in Proceedings of the 4th IEEE ComSoc International Workshop on Multimedia Communications (MULTIMEDIA '92), pp. 40-56, Monterey, Calif, USA, April 1992.

[19] S.-F. Chang and D. G. Messerschmitt, "Manipulation and compositing of MC-DCT compressed video," IEEE Journal on Selected Areas in Communications, vol. 13, no. 1, part 2, pp. 1-11, 1995.

[20] Y. Noguchi, D. G. Messerschmitt, and S.-F. Chang, "MPEG video compositing in the compressed domain," in Proceedings of IEEE International Symposium on Circuits and Systems (ISCAS '96), vol. 2, pp. 596-599, Atlanta, Ga, USA, May 1996.

[21] B. Yu and K. Nahrstedt, "Internet-based interactive HDTV," Multimedia Systems, vol. 9, no. 5, pp. 477-489, 2004.

[22] Y.-P. Tan and H. Sun, "Fast motion re-estimation for arbitrary downsizing video transcoding using H.264/AVC standard," IEEE Transactions on Consumer Electronics, vol. 50, no. 3, pp. 887-894, 2004.

[23] C.-H. Li, C.-N. Wang, and T. Chiang, "A fast downsizing video transcoding based on H.264/AVC standard," in Proceedings of the 5th IEEE Pacific Rim Conference on Multimedia (PCM '04), pp. 215-223, Tokyo, Japan, November-December 2004.

[24] H. Shen, X. Sun, F. Wu, H. Li, and S. Li, "A fast downsizing video transcoder for H.264/AVC with rate-distortion optimal mode decision," in Proceedings of IEEE International Conference on Multimedia and Expo (ICME '06), vol. 1, pp. 20172020, Toronto, Ontario, Canada, July 2006.

[25] N. Merhav and V. Bhaskaran, "A fast algorithm for DCTdomain inverse motion compensation," in Proceedings of IEEE International Conference on Acoustics, Speech, and Signal Processing (ICASSP '96), vol. 4, pp. 2307-2310, Atlanta, Ga, USA, May 1996.
[26] J. Song and B.-L. Yeo, "A fast algorithm for DCT-domain inverse motion compensation based on shared information in a macroblock," IEEE Transactions on Circuits and Systems for Video Technology, vol. 10, no. 5, pp. 767-775, 2000.

[27] S. Liu and A. C. Bovik, "Local bandwidth constrained fast inverse motion compensation for DCT-domain video transcoding," IEEE Transactions on Circuits and Systems for Video Technology, vol. 12, no. 5, pp. 309-319, 2002.

[28] H. Shen, X. Sun, F. Wu, H. Li, and S. Li, "A fast downsizing video transcoder for H.264/AVC with rate-distortion optimal mode decision," in Proceedings of IEEE International Conference on Multimedia and Expo (ICME '06), pp. 2017-2020, Toronto, Ontario, Canada, July 2006.

[29] J. Bialkowski, M. Barkouwsky, F. Leschka, and A. Kaup, "Lowcomplexity transcoding of inter coded video frames from H.264 to H.263," in Proceedings of IEEE International Conference on Image Processing (ICIP '06), pp. 837-840, Atlanta, Ga, USA, October 2006.

[30] J. H. Hur and Y. L. Lee, "H.264 to MPEG-4 transcoding using block type information," in Proceedings of IEEE International Conference Region 10 (TENCON '05), pp. 1-6, Melbourne, Australia, November 2005.

[31] Y.-P. Tan and H. Sun, "Fast motion re-estimation for arbitrary downsizing video transcoding using H.264/AVC standard," IEEE Transactions on Consumer Electronics, vol. 50, no. 3, pp. 887-894, 2004.

[32] P. Zhang, Y. Lu, Q. Huang, and W. Gao, "Mode mapping method for H.264/AVC spatial downscaling transcoding," in Proceedings of International Conference on Image Processing (ICIP '04), vol. 4, pp. 2781-2784, Singapore, October 2004.

[33] I.-H. Shin, Y.-L. Lee, and H.-W. Park, "Motion estimation for frame-rate reduction in H.264 transcoding," in Proceedings of the 2nd IEEE Workshop on Software Technologies for Future Embedded and Ubiquitous Systems (WSTFES '04), vol. 4, pp. 6367, Vienna, Austria, May 2004.

[34] D. Lefol and D. Bull, "Mode refinement algorithm for H.264 inter frame requantization," in Proceedings of IEEE International Conference on Image Processing (ICIP '06), pp. 845-848, Atlanta, Ga, USA, October 2006.

[35] J. Zhang, A. Perkis, and N. D. Georganas, "H.264/AVC and transcoding for multimedia adaptation," in Proceedings of the 6th COST 276 Workshop, Thessaloniki, Greece, May 2004.

[36] X. Xiu, L. Zhuo, and L. Shen, "A H.264 bit rate transcoding scheme based on PID controller," in Proceedings of IEEE International Symposium on Communications and Information Technologies (ISCIT'05), vol. 2, pp. 1074-1077, Beijing, China, October 2005.

[37] D. Lefol, D. Bull, and N. Canagarajah, "Performance evaluation of transcoding algorithms for H.264," IEEE Transactions on Consumer Electronics, vol. 52, no. 1, pp. 215-222, 2006.

[38] C.-H. Li, C.-N. Wang, and T. Chiang, "A low complexity picture-in-picture transcoder for video-on-demand," in Proceedings of IEEE International Conference on Wireless Networks, Communications and Mobile Computing (WirelessCom '05), vol. 2, pp. 1382-1387, Maui, Hawaii, USA, June 2005.

[39] C.-H. Li, H. Lin, C.-N. Wang, and T. Chiang, "A fast H.264based picture-in-picture (PIP) transcoder," in Proceedings of IEEE International Conference on Multimedia and Expo (ICME '04), vol. 3, pp. 1691-1694, Taipei, Taiwan, June 2004.

[40] A. Levi and H. Stark, "Restoration from phase and magnitude by generalized projections," in Image Recovery Theory and Application, pp. 277-319, Academic Press, Orlando, Fla, USA, 1987. 
[41] S.-H. Wang, W.-H. Peng, Y. He, et al., "A software-hardware co-implementation of MPEG-4 advanced video coding (AVC) decoder with block level pipelining," The Journal of VLSI Signal Processing, vol. 41, no. 1, pp. 93-110, 2005.

[42] C. Chen, P.-H. Wu, and H. Chen, "Transform-domain intra prediction for H.264," in Proceedings of IEEE International Symposium on Circuits and Systems (ISCAS '05), vol. 2, pp. 1497-1500, Kobe, Japan, May 2005.

Chih-Hung Li was born in Taipei, Taiwan in 1979. He received the B.S. degree in electronics engineering from National ChiaoTung University, Hsinchu, Taiwan, in 2001, where he is currently pursuing his Ph.D. degree in the Institute of Electronics. His research interests include video transcoding, platform-based SoC design, system architecture modeling, and DRAM controller.

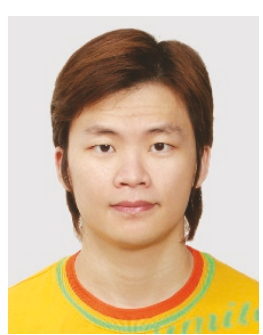

Chung-Neng Wang was born in PingTung, Taiwan, in 1972. He received the B.S. degree and the Ph.D. degree in computer science and information engineering from National Chiao-Tung University (NCTU), HsinChu, Taiwan, in 1994 and 2003, respectively. He joined the faculty at National Chiao-Tung University, in Taiwan, in January 2003. Since 2001, he has actively participated in ISO's Moving Picture Experts

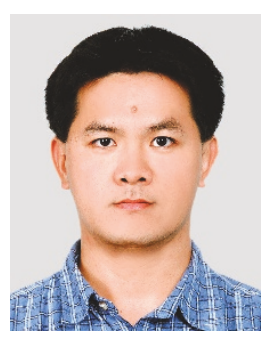
Group (MPEG) digital video coding standardization process. He has made more than 50 contributions to the MPEG committee over the past 4 years. He published over 36 technical journal and conference papers in the field of video and signal processing. His current research interests are video/image compression, motion estimation, video transcoding, and streaming.

Tihao Chiang was born in Cha-Yi, Taiwan, Republic of China, in 1965. He received the B.S. degree in electrical engineering from the National Taiwan University, Taipei, Taiwan, in 1987, and the M.S. and Ph.D. degrees in electrical engineering from Columbia University in 1991 and 1995. In 1995, he joined David Sarnoff Research Center (formerly RCA laboratory) as a Member of Technical Staff. Later, he was

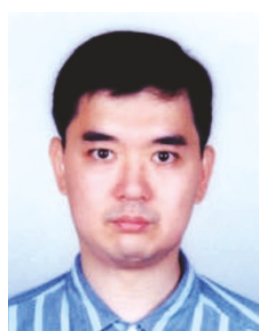
later promoted as a technology leader and a program manager at Sarnoff. For his work in the encoder and MPEG-4 areas, he received two Sarnoff achievement awards and three Sarnoff team awards. Since 1992 he has actively participated in ISO's Moving Picture Experts Group (MPEG) digital video coding standardization process and has made more than 100 contributions to the MPEG committee over the past 15 years. In September 1999, he joined the faculty at National Chiao-Tung University in Taiwan, Republic of China On his sabbatical leave from 2004, he worked with Ambarella USA and initiated its R\&D operation in Taiwan. Dr. Chiang is currently a Senior Member of IEEE and holder of over 40 patents. He published over 70 technical journal and conference papers in the field of video and signal processing. 